\title{
Measurement of Wave Fronts Without a Reference Stand- ard: Part 2. The Wave Front-Reversing Interferometer
}

\author{
J. B. Saunders
}

(November 28, 1961)

\begin{abstract}
Interferometers permitting the measurement of shape and altered distribution of fringes are usually used to compare either an unknown wave front with a known one or an unknown surface with a standard reference surface. Any error in the reference surface introduces a corresponding error in the results sought. This paper describes an absolute measuring interferometer and the associated mathematical operations necessary for analysis of the data. The reference surface is purely mathematical in nature and is therefore free from error. Deviations of converging wave fronts from perfect spheres, paraboloids or ellipsoids are readily measured without the use of tangible reference surfaces.

The equation of the reference surface may contain one or more parameters whose value is sought. Thus, the eccentricity of the conicoid that best fits a mirror and deviations of the surface from the true mathematical curve are obtained simultaneously. The sensitivity of the test can be varied so that when testing large aspherical elements which depart very far from spheres the number and width of the fringes can be adjusted to any desired values.
\end{abstract}

\section{Introduction}

Many tests made by interferometry consist in measuring the variation in separation of two wave fronts. Usually, the shape of one of the wave fronts is known so that the variation in the observed separation is a measure of the absolute shape of the unknown. The shape of the wave front is a function of the optical elements (lenses, mirrors, etc.) that produce it. Consequently, the aberrations of lenses, the shapes of mirror surfaces, the variations in densities of fluids, etc., are obtainable from the measurement of wave-front shapes.

Usually the known wave front is produced by a mirror of know shape called the reference surface and the unknown wave front which is produced by the specimen is compared with it. If the reference surface is imperfect, any error in ic is reflected into, or transferred to, the measured shape of the unknown.

This paper describes a method of comparing wave fronts against a reference surface which is purely mathematical in nature. Since the Koesters doubleimage prism, which is the basic element of this interferometer, can be made with a high degree of perfection [1] ${ }^{1}$ the results are practically free from instrumental error and the necessity for making reference mirrors and lenses of various foci is eliminnated. The interferometer is quite stable, compact, and is easily operated by nontechnical personnel.

This method of analyzing interferometer data, with the necessary modifications required to adapt it to the wave-front-shearing interferometer (三WSI), has been described in part 1 of this series of papers [2].

1 Figures in brackets indicate the literature references at the end of this paper.
Without this development, neither the WSI nor the wave front reversing interferometer (三WRI), will yield unique results except when applied to wave fronts which are known (from some other type of test or source of information) to have revolution symmetry.

\section{Description of the Method}

To describe the principles used in this method of analysis its application to the testing of a simple lens will be used for illustration. The operations described here apply, without change, to the testing of lenses by autocollimation (one conjugate at infinity), the testing of mirrors, and to all other tests where the results sought can be obtained from a knowledge of wave-front shapes. This treatment is applicable to three dimensional space but for simplification the mathematics will be limited to a plane.

\subsection{Symbols and Abbreviations}

The discussions and treatment of this problem require a considerable number of symbols. To provide a ready reference, all quantities used are defined below.

$a=$ diameter of the circular aperture of the lens or mirror under test.

$B=$ dividing plane of the double-image prism.

$C$ and $C^{\prime}=$ images of conic section.

$C_{1}, C_{2}$, and $C_{2}^{\prime}=$ arcs of reference curves representing known wave fronts; (i.e., ares of circles if a corrected lens or spherical mirror is considered and ares of a conic section if a comcoid is being considered).

$D=$ position of small, monochromatic light source.

$E=$ center of curvature of prism base. $e=$ eccentricity of conic section. 
$F=$ ideal image point (conjugate of $D$ in fig. 1).

$F_{1}, F_{2}, F^{\prime}=$ images of $F\left(F_{1}\right.$ and $F_{2}$ are imaged by the outer plane surfaces of the prism and $F^{\prime}$ is imaged by the dividing plane $B$.)

$F_{c}$ and $\mathrm{F}_{c}^{\prime}=$ centers of curvature of conic section corresponding to points of extreme curvature.

$h$ and $k=$ coordinates of $F_{c}^{\prime}$.

$h$ and $k=$ components of the line from $F$ to $E$, perpendicular and parallel to the dividing plane, respectively.

$N_{r \sigma}=$ observed order of interference at $P_{p}$.

$P_{\nu}$ and $P_{\sigma}=$ any chosen pair of superimposed reference points. (These points are equally distant [optically] from the light source and, therefore, fall on the same wave front, Their positions on the wave front are indicated to a sufficient approximation by spots on the lens surface, by beads on a thin wire or string, or by lines on a scale.)

$R=$ radius of curvature of a conic section minimum for $e^{2}$ positive and maximum for $e^{2}$ negative.

$S=$ separation of reference curves $C_{1}$ and $C_{2}^{\prime}$ (or $C$ and $C^{\prime}$ ) along the line of sight from $E$ through $P_{\nu}$.

$T, T^{\prime}, T_{1}, T_{1}^{\prime}, T_{2}, T_{2}^{\prime}=$ reference points on a conic section.

$u$ and $v=$ coordinates of $E$.

$V$ and $V^{\prime}=$ perihelion of conic section.

$W, W_{1}, W_{2}$, and $W_{2}^{\prime}=$ respectively. the wave front before it is incident on the lens or mirror, the part of the transmitted wave front that lies above the dividing plane, the transmitted part that lies below the dividing plane and the image of $W_{2}$ with respect to the dividing plane.

$\mathrm{WSI}=$ wave-front-shearing interferometer

$\mathrm{WRI}=$ wave-front-reversing interterometer .

$x$ and $y=$ coordinates of $T$.

$\alpha=$ angle between the dividing plane and the optical axis.

$\beta=$ angle between the dividing plane and the radius vector to $\rho_{y}$.

$\delta_{\nu}=$ separations between $C_{1}$ and $W_{1}$ along the radius vector to $\rho_{\nu}$

$\delta_{\sigma}=$ separation between $C_{2}$ and $W_{2}$ along the radius vector to $P_{\sigma}$, (or separation between $C_{2}^{\prime}$ and $W_{2}^{\prime}$ along the radius vector to $P_{\nu}$ ).

$\epsilon=$ angle between two images of conic section.

$\epsilon_{\mu}=$ angle between $C_{1}$ and $C_{2}^{\prime}$ at their point of intersection with the dividing plane.

$\lambda=$ wavelength of light

$\mu=$ distance from the extension of the dividing plane to the axial center of the lens (fig. 1). It is positive when the dividing plane is above the axis of the lens and negative when below.

$\nu$ and $\sigma=$ distances from $P_{\nu}$ and $P_{\sigma}$, respectively, to the optical axis. These distances are measured along the arc of the reference curve, $C_{1} C_{2}$. They are positive when the corresponding points are above the axis of the lens and negative when below.

$\rho, \rho^{\prime}, \rho_{c 1}$, and $\rho_{c 2}=$ radius vectors from $E$ to $T, T^{\prime}, T_{1}$, and $T_{2}$, respectively

$\rho_{\nu}$ and $\rho_{\sigma}=$ radius vectors from $E$ to $P_{v}$ and $P_{\sigma}$. respectively.

$\phi=$ the sum of the squares of the deviations (defined by eq (13)).

\subsection{Apparatus}

A simple interferometer for testing lenses, without a reference surface or element for comparison, is shown in figure 1. It consists of a small monochromatic light source at $D$, the lens to be tested and a double-image prism [1]. The lens received the diverging wave front, $W$ (spherical when three dimensional space is considered and circular for two dimensional space) and changes it into a converging wave front. If the lens were perfectly corrected for the indicated position of the source, the converging wave front would be an arc of a circle (curve $C_{1} C_{2}$ ) and the light would form a perfect image (airy disk) of the source at the ideal image point $F$. The wave front produced by an imperfect lens (greatly exaggerated) is indicated by curve $W_{1} W_{2}$ and its deviation from the ideal wave front, $C_{1} C_{2}$, is a measure of the abberations of the lens. The circle that represents the ideal wave front exists in mathematical form only and its parameters are adjusted statistically or otherwise chosen so that it fits the real wave front in accordance with some arbitrarily selected specification. Methods of choosing the reference circle will be described later.

In practice the prism is mounted so as to pivot about $E$, and $E$ is placed near $F$. The semi-reflecting dividing plane, $B$, is adjusted so that its extension cuts the circular lens along a chord at a chosen distance, $\mu$, from the axis of the Jens. Each new adjustment of the prism (change in value of $\mu$ ) introduces and additional arbitrary value for $\epsilon_{\mu}$. The quantity $\mu$ is positive when the dividing plane is above the axis of the lens and negative when below.

The effect produced by this prism on the wavefront is as follows: the wave front $W$, after division by wave-front division into two parts, $W_{1}$ above the dividing plane and $W_{2}$ below, is further subdivided by amplitude division at $B$ into two beams each, making four beams altogether, two arriving at each of the points $F_{1}$ and $F_{2}$. One of the beams that arrives at each of these points suffers two reflections and the other a single reflection. Consequently, a mirror image of everything seen below the dividing plane appears above it and vice versa. The arc $C_{2}$ (fig. $2 \mathrm{~A}$ ) appears as $C_{2}{ }^{\prime}$. The center of curvature of $C_{2}$, [3] located at $F$, is imaged at $F^{\prime}$ which is the center of curvature of $C_{2}^{\prime}$. The part $W_{2}$ of the wave front below the dividing plane appears as $W_{2}{ }^{\prime}$, so that $W_{1}$ and $W_{2}$ appear inverted with respect to each other and superimposed in the overlapping region $W_{2}^{\prime}$, where fringes of interference (fig. 2B) are observed.

The two halves of the prism are slightly rotated about an axis normal to the dividing plane (as described in references 1 and 6). A wave front having symmetry with respect to the dividing plane would therefore give rise to straight fringes normal to the line representing the dividing plane in the field of view. If the two halves of the prism are not rotated relative to each other the fringes are straight and parallel to the dividing plane.

\subsection{Equations of Observation}

The separation of wave fronts, $W_{1}$ and $W_{2}^{\prime}$ (fig. $2 \mathrm{~A}$ ), is the optical path difference $\rho_{\nu}-\rho_{\sigma}$ which is 


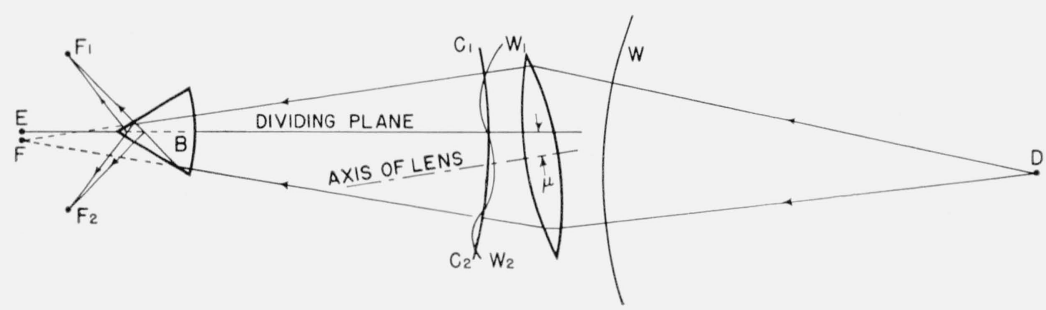

Figure 1. Optical arrangement for testing a lens for a finite object distance. This arrangement requires the use of polarized light because of polarized effects in the prism.

Figure 2. A. Illustration of image formation by the double-image prism.

B. Interference fringes obtained with the assembly of figure 1.

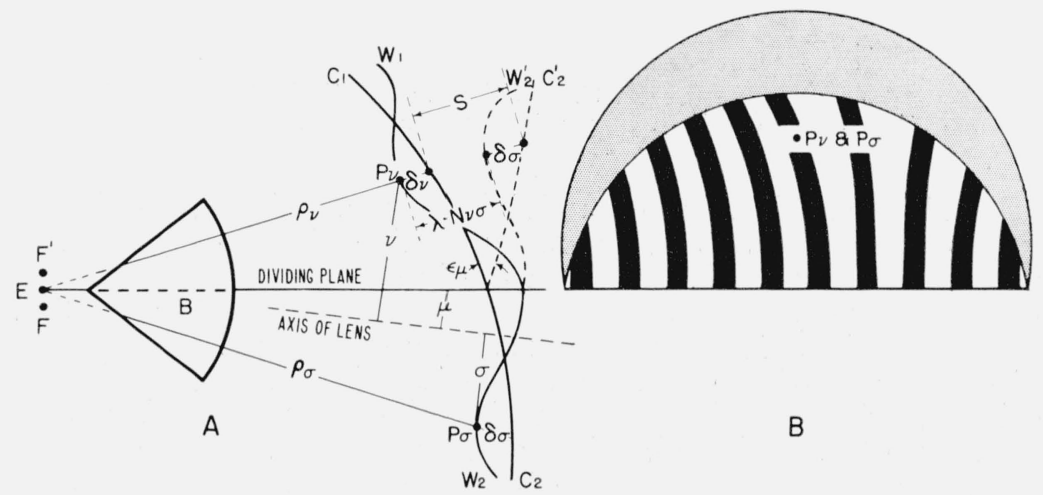

equal to the product $\lambda N_{\nu \sigma}$. The corresponding separation of the reference circle $C_{1}$ and $C_{2}{ }^{\prime}$ at $P_{\nu}$ is, approximately [2].

$$
S=(\nu-\mu) \epsilon_{\mu}=\epsilon_{\mu}(\mu-\sigma) .
$$

The two quantities in parentheses are the distances, along the circle, from $P_{\nu}$ and $P_{\sigma}$, respectively, to the dividing plane. Since $\mathrm{P}_{\sigma}$, in figure 2 , is below the axis of the lens, $\sigma$ is a negative quantity.

It is apparent from figure $2 \mathrm{~A}$ that the relation between the four separations, $\delta_{\nu}, \delta_{\sigma} S$, and $\lambda N_{\nu \sigma}$ is given by the equation

$$
\delta_{\nu}+S=\delta_{\sigma}+\lambda N_{\nu \sigma} .
$$

See section 2.1 for definitions of these quantities. On substituting for $S$ its value from eq (1) and transferring terms we have

$$
\delta_{\nu}-\delta_{\sigma}=\lambda N_{\nu \sigma}-\epsilon_{\mu}(\nu-\mu)
$$

which are the basic equations of observation, providing relationships between the deviations of the wave front from the abstract curve $C_{1} C_{2}$.

\subsection{Distribution and Number of Reference Points}

If the surfaces of the lens are figures of revolution, the optic axis is well defined, and can be accurately located by observing the fringe pattern while changing the value of $\mu$. When the dividing plane coincides with the optic axis the fringes will be straight regardless of aberrations in the lens. If the lens surfaces are not figures of revolution the changes in curvature and spacing of the fringes will indicate the most probable position of the axis.

The reference points are chosen in the aperture of the lens, along a straight line normal to the dividing plane of the prism and through the optical axis, regardless of whether the axis is or is not centered in the aperture of the lens. The positions of these points are measured from the optical axis. They are positive when above and negative when below the axis. The points are equally spaced and their separation is defined as unity. An odd or an even number of reference points, symmetrically placed above and below the axis, may be used An odd set, therefore, would be located at integral units of length from the axis, as in figure $3-\mathrm{A}$, while an even set would be placed at odd integral half units of length from the axis, as shown in figure $3-\mathrm{B}$. If a point at the center is desired one must choose an odd number of points. Figure 3-C illustrates the arrangement of an odd set when the optic axis is not centered in the aperture. The interferometer is sufficiently sensitive to detect deviations of the position of the axis from the center of the aperture in most lenses.

Any number of points may be selected along the principal diameter (the diameter that is normal to the dividing plane) by adjusting the separation of adjacent points. If the mirror or lens to be tested is unsymmetrical and/or irregular the test should be made with more points than when a smooth wave front is formed.

Even when the wave front is smooth and symmetrical about the axis, many reference points will sometimes be required. The number of fringes in the field of view depends upon three quantities. These are: (a) The magnitude of the wedge that is built into the prism, (b) the angle between the wave fronts at the dividing plane, and (c) the relative shapes of the two images, $W_{1}$ and $W_{2}{ }^{\prime}$, of the wave front. The effects of these three quantities are more fully described as follows:

(a) The built-in wedge of the prism produces an invariant wedge between the wave-front images. Its 


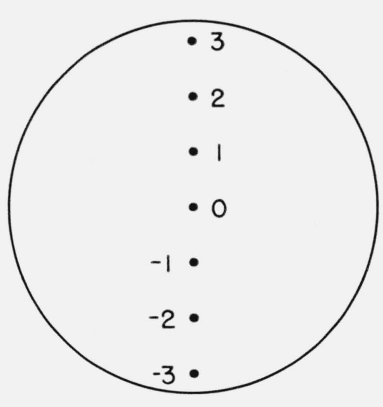

A

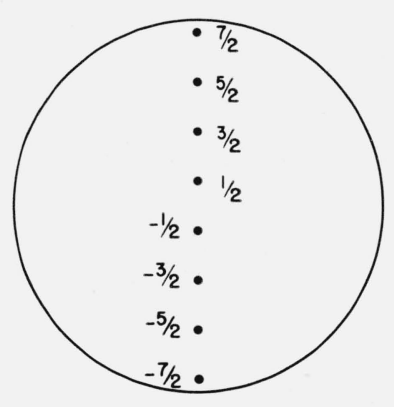

B

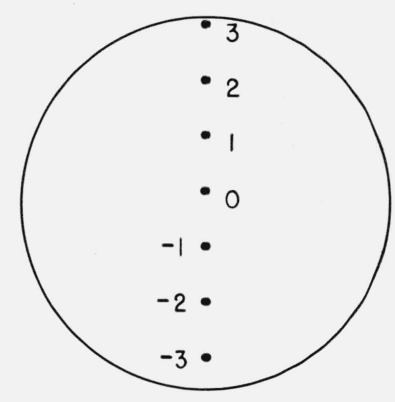

C

Figure 3. $A, B$, and $C$ are, respectively, the distribution of the reference points when the number is odd, when the number is even, and when the optic axis is not centered in the lens.

vertex is perpendicular to the dividing plane. Since the component of this wedge, along the line of reference points, is zero, its effect on the interference at the reference points is to add a constant to the observed orders of interference at all reference points. If the vertex of the built-in wedge is adjusted to coincide with the line along which the reference points are to be selected, this wedge does not affect the order of interference at the reference points. It does, however, ensure that the fringes never get too broad for precision readings.

(b) The angle, $\epsilon_{\mu}$, between the wave fronts at the dividing plane is equal to the angle between lines drawn from points $F^{\prime}$ and $F^{\prime}$ to the vertex of this angle (see fig. 13, A). This angle is adjustable by varying the position of $F$ relative to $E$, by displacing the prism laterally. Thus the component of fringe widths perpendicular to the dividing plane and the order of interference at any chosen reference point can be adjusted to any desired value. If the two wave fronts, $W_{1}$ and $W_{2}^{\prime}$, are identical in shape (i.e., if wave front $W_{1} W_{2}$ is spherical) the difference in order of interference between all pairs of adjacent points is approximately constant and adjustable at will.

(c) If the wave front $W_{1} W_{2}$ is not spherical the two images of it, $W_{1}$ and $W_{2}^{\prime}$, will not be identical unless $W_{1} W_{2}$ has revolution symmetry and the value of $\mu$ is zero. When testing a wave front that departs very far from a sphere, as, for instance, when testing an $f / 1$ parabola of large aperture, the minimum number of fringes that can be obtained by adjusting $\epsilon_{\mu}$ depends upon $\mu$. Since, in operation of the instrument, pairs of points are brought into coincidence for conditions other than $\mu=0$, and since the absolute magnitude of $\mu$ depends upon the unit separation of points, it follows that the choice of the number of reference points determines the minimum attainable number of fringes. If the number of reference points is too small the minimum attainable number of fringes may be too large for accurate readings. Consequently, a large number of points may be required to obtain the optimum reading condition. Since the sensivity of the interferometer depends upon the ratio $\mu / a$, the sensivity also depends upon the number of reference points. The variation of sensivity with the ratio $\mu / a$ and its effects on the required number of reference points are fully explained under "Sensitivity" in part 1 of this series [2].

\subsection{Solution of Wave-Front Deviations}

We shall select (fig. 4) quite arbitrarily for this discussion, seven reference points along the principal diameter of the lens and with their spacings subtending equal angles at $E$. These points will be considered as lying on a common wave front at the time it emerges from the Jens, and their separations will be defined as one unit of length. Since the points are observed from position $E$ they may be indicated with small beads on a fine wire that is stretched across the face of the lens (fig. $4 \mathrm{~A}$ ).

If the dividing plane is adjusted to include one of the inner points (any point except an end one) as in figure $4 \mathrm{C}$, some of the points will appear to coincide in pairs. If it is adjusted to bisect the angle between any two of the inner points, as in figure $4 \mathrm{~B}$, some of the points again appear to coincide in pairs. The points will coincide in pairs when $\mu$ is an integral number of half units of length but will not coincide for other values of $\mu$.

We may substitute, in eq (3), the observed values for $N_{\nu \sigma}$ and $(\nu-\mu)$ at each pair of points, successively. This yields as many equations of observation as there are pairs of coinciding points. We may then change the value of $\mu$, thereby changing the combination of paired points, and obtain additional equations. Each new value for $\mu$ (i.e., $\mu=0, \pm 0.5$ $\pm 1.0, \ldots$. . introduces several additional equations but only one additional unknown-i.e., a new value for $\epsilon_{\mu}$. In this manner, we can obtain more equations than we have unknowns; and it would seem that a statistical analysis of these equations would permit an evaluation of the several deviations (values for the $\delta$ 's). However, such is not the case. The relation between these equations are such that the resultant number of independent equations is always less by three than the number of unknowns.

When the wave front is completely unknown, the minimum number of observation equations that permit a unique solution of the $\delta$ 's require two sets of fringes (two values for $\mu$ ). Each adjustment of the interferometer introduces a new value for $\epsilon_{\mu}$ because the value of $\epsilon_{\mu}$ can not be retained from 


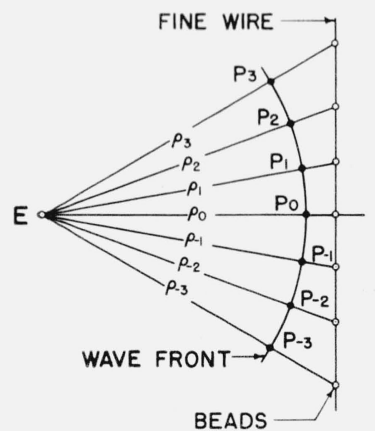

A

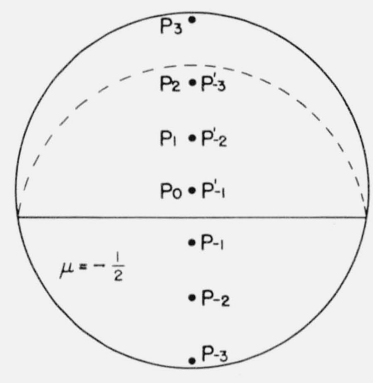

B

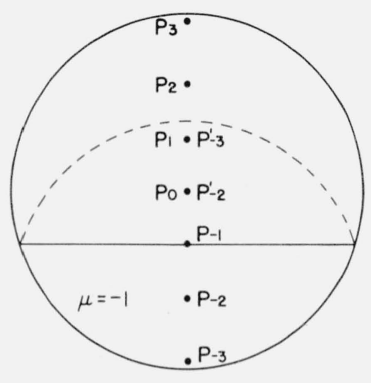

C

FrGURE 4. A. Illustration to show the manner of locating the reference points, $\mathrm{P} \nu$, by means of beads on a fine wire.

B. and C. Distribution of points and images of points for $\mu=-0.5$ and $\mu=-1$, respectively.

one adjustment to another. For simplicity we will use the two adjustments ( 2 sets of fringes) that are obtained with $\mu=0$ and $\mu=0.5$. These are (for the 7 chosen reference points) for $\mu=0$

and for $\mu=0.5$

$$
\begin{aligned}
& \delta_{1}=\delta_{-1}+\lambda N_{1,-1}-\epsilon_{0}, \\
& \delta_{2}=\delta_{-2}+\lambda N_{2,-2}-2 \epsilon_{0}, \\
& \delta_{3}=\delta_{-3}+\lambda N_{3,-3}-3 \epsilon_{0},
\end{aligned}
$$

$$
\delta_{1}=\delta_{0}+\lambda N_{1,0}-0.5 \epsilon_{0.5}
$$$$
\delta_{2}=\delta_{-1}+\lambda N_{2,-1}-1.5 \epsilon_{0.5} \text {, }
$$

$$
\delta_{3}=\delta_{-2}+\lambda N_{3,-2}-2.5 \epsilon_{0.5} \text {. }
$$

Equations 4 through 9, form a set of 6 simultaneous equations having 9 unknowns (7 $\delta$ 's and $2 \epsilon_{\mu}^{\prime}$ 's); consequently, we either have an excess of 3 unknowns or a shortage of 3 equations for obtaining a unique solution. It is quite obvious that we cannot evaluate deviations from an unidentified curve. The persistent shortage of three equations is associated with the three conditions necessary to define an arbitrarily chosen 3-parameter reference circle.

The method used in combining the three condition equations with the set of observation equations is fully described under the heading "Specifications for the Reference Circle" that appears in part 1[2]. Since the details of the application to the WRI are so similar to those given for the WSI, this treatment will be limited to details that are necessary for a clear understanding of the WRI. Since three points fully define a circle we may define the reference circle, $C_{1} C_{2}$, as that particular circle which passes through any three of the chosen points, by equating the corresponding $\delta$ 's to zero. Thus, if we impose the three conditions,

$$
\delta_{3}=\delta_{0}=\delta_{-3}=0
$$

we are requiring that the reference circle shall pass through points $P_{3}, P_{0}$, and $P_{-3}$, as indicated in figure $5 \mathrm{~B}$. This may be considered as either a reduction in the number of unknowns by three or an increase of three in the number of independent simultaneous equations. In either case we get as many equations as unknowns so that a unique solution of all unknowns is possible. Any three of the chosen reference points may have been selected to fall on the circle.
The simplest manner of specifying and computing the reference circle (called the "Method of Coincidence") may not produce a good fitting circle; but this does not render any less obvious the location of the high and low places on the wave front. Consequently, an optical worker would have no trouble locating the high and low places for further polishing to reduce the deviations.

A better fitting reference circle is obtained by applying the method of averages [4]. Figure 5C shows the same wave front with a circle of reference that is determined by this method. The method of averages allows a variety of groupings of points and assignments of weights. For instance, since the areas of the wave fronts that are associated with the several points increases with $\nu$ we might multiply all $\delta$ 's in eq (10) by the quantity $(\nu+1)$ so as to assign unit weight to the axial point, $P_{0}$, and corresponding higher weights to other points, thus distributing the weight more nearly proportional to the areas of the several zones.

A good fit, based on the method of averages, is obtained by imposing the following simple conditions :

$$
\left.\begin{array}{l}
\delta_{3}+\delta_{2}=0 \\
\delta_{1}+\delta_{0}+\delta_{-1}=0 \\
\delta_{-2}+\delta_{-3}=0 .
\end{array}\right\}
$$

The circle that fits the wave front shown in figure 5 , in accordance with the conditions of eq (11) is shown in figure 5C. Equations (11), when added to the six equations of observation (eq (4) through (9)), again supply the necessary additional independent equations for a complete solution of all $\delta$ 's.

The best fitting circle, shown in figure $5 \mathrm{D}$, is defined by a third set of three conditions,

$$
\frac{\partial \phi}{\partial \delta_{0}}=\frac{\partial \phi}{\partial \epsilon_{0}}=\frac{\partial \phi}{\partial \epsilon_{0.5}}=0
$$

where

$$
\phi \equiv \sum_{\nu=-3}^{3} \delta_{\nu}^{2}=\delta_{-3}^{2}+\delta_{-2}^{2}+\delta_{-1}^{2}+\delta_{0}^{2}+\delta_{1}^{2}+\delta_{2}^{2}+\delta_{3}^{2}
$$




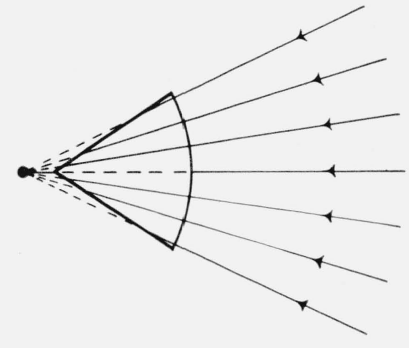

A

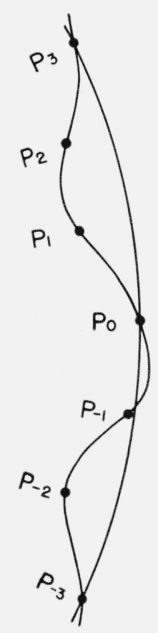

B

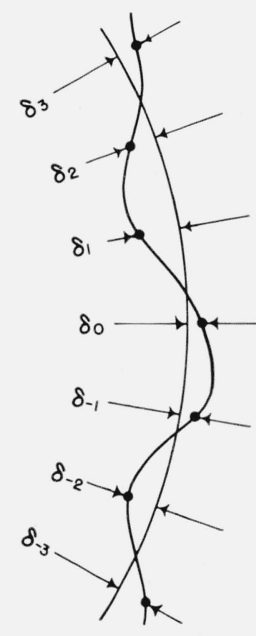

C

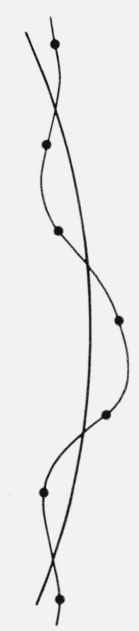

D

FIGURE 5. A. Illustration to show the distribution of the reference points relative to the prism.

$\mathrm{B}, \mathrm{C}$, and $\mathrm{D}$. The positions of the reference circles relative to the wave front when the reference circle is chosen in accordance with the method of coincidence, the method of averciges, and the method of least squares, respectively.

which requires that the sum of the squares of the deviations be a minimum. Differentiation with respect to any other three of the nine unknowns (i.e., $7 \delta$ 's and $2 \epsilon_{\mu}$ 's) will yield the same set of condition equations.

In order to determine the equations of condition corresponding to eq (12), it will be convenient to solve for the $\delta_{\nu}$ 's. These are:

$$
\begin{array}{ll}
\delta_{0}=\delta_{0} & \text { (obvious) } \\
\left.\delta_{1}=\delta_{0}+\lambda N_{1,0}-0.5 \epsilon_{0.5} \text { (from } 7\right) &
\end{array}
$$

$\delta_{-1}=\delta_{0}+\lambda\left(N_{1,0}-N_{1,-1}\right)-0.5 \epsilon_{0.5}+\epsilon_{0}($ from 4 and 15$)$

$$
\begin{aligned}
\delta_{2}=\delta_{0}+\lambda\left(N_{1,0}-\right. & \left.N_{1,-1}+N_{2,-1}\right) \\
& -2.0 \epsilon_{0.5}+\epsilon_{0} \text { (from } 8 \text { and } 16 \text { ) }
\end{aligned}
$$

$\delta_{-2}=\delta_{0}+\lambda\left(N_{1,0}-N_{1,-1}+N_{2,-1}-N_{2,-2}\right)$

$$
-2.0 \epsilon_{0.5}+3 \epsilon_{0} \text { (from } 5 \text { and } 17 \text { ) }
$$

$$
\begin{array}{r}
\delta_{3}=\delta_{0}+\lambda\left(N_{1,0}-N_{1,-1}+N_{2,-1}-N_{2,-2}+N_{3,-2}\right) \\
-4.5 \epsilon_{0.5}+3 \epsilon_{0}(\text { from } 9 \text { and } 18)
\end{array}
$$

$\delta_{-3}=\delta_{0}+\lambda\left(N_{1,0}-N_{1,-1}+N_{2,-1}-N_{2,-2}+N_{3,-2}-N_{3,-3}\right)$ $-4.5 \epsilon_{0.5}+6 \epsilon_{0}$ (from 6 and 19 )

On performing the differentiations indicated in eq (12), and substituting the corresponding values for these differentials from eqs (14) through (20), we obtain the set of condition equations for the method of least squares. These are:

$$
\left.\begin{array}{l}
\delta_{3}+\delta_{2}+\delta_{1}+\delta_{0}+\delta_{-1}+\delta_{-2}+\delta_{-3}=0 \\
\delta_{-1}+\delta_{2}+3\left(\delta_{-2}+\delta_{3}\right)+6 \delta_{-3}=0 . \\
\left(\delta_{1}+\delta_{-1}\right)+4\left(\delta_{2}+\delta_{-2}\right)+9\left(\delta_{3}+\delta_{-3}\right)=0
\end{array}\right\}
$$

Any one of the sets of conditional equations (given by eqs $(10,(11)$, or (21) combined with the observation equations (4) through (9)) permits a complete solution of all unknowns. The operations that lead to a solution of these equations are performed as follows: (1) By adjusting the interferometer so that $\mu=0$ (i.e., with the dividing plane cutting the wave front along a line through the axis of the lens; (2) substituting the observed values for $(\nu-\mu)$ and the observed orders of interference at each of the three pairs of coinciding points into eq (3) thus obtaining eqs (4), (5), and (6): (3) readjusting $\mu$ to equal 0.5 (fig. $4 \mathrm{~B}$ ): (4) again substituting the observed quantities $N_{\nu \sigma}, \nu$ and $\mu$ into eq (3), thus obtaining eqs (7), (8), and (9): (5) solving for the 9 unknowns in the 9 linear, simultaneous equations (eqs (4) through (9) and any one of (10), (11), or (21)).

\subsection{Symmetrical Systems}

Lenses and mirrors that have been polished mechanically produce symmetrical wave fronts when tested on axis. For such symmetrical elements all $\delta$ 's on one side of the axis of symmetry are equal to corresponding $\delta$ 's on the other side and for $\mu=0$ 
the fringes of interference will be straight. All $\delta$ 's with negative subscripts can, therefore, be replaced with the corresponding positive subscripts. In the case of seven reference points, chosen here for illustration, the left hand side of eqs (4), (5), and (6) becomes zero and the right hand side of each of these equations represents a solution of $\epsilon_{0}$. Since $\epsilon_{0}$ is superfluous, the adjustment of $\mu$ to zero for a symmetrical wave front serves only as a check on symmetry. Consequently, if symmetry is observed, eqs (4), (5), and (6) are exempted from the group of simultaneous equations. The elimination of negative subscripts reduces the number of unknowns so that a single adjustment $(\mu=0.5$, eqs $(7),(8)$ and (9) of the interferometer is sufficient for a complete solution. Equations (4), (5), and (6) are eliminated and eqs $(10,(11)$, and (12) for the three methods, are reduced, respectively, to $\delta_{3}=$

$\delta_{0}=0, \delta_{3}+\delta_{2}=2 \delta_{1}+\delta_{0}=0$, and $\frac{\partial \phi}{\partial \epsilon_{0.5}}=\frac{\partial \phi}{\partial \delta_{0}}=0$.

The number of equations in the complete set is reduced from 9 to 5 with a corresponding reduction in the number of unknowns.

\subsection{Illustration}

The process described above is illustrated in the following application to the testing of a 12 -in. aperture, $15-\mathrm{ft}$ focal length astronomical refractor. Figure 6 shows the optical arrangement for testing with one conjugate at infinity. Figure $7 \mathrm{~A}$ is a photograph of the fringes taken with the dividing plane coinciding with the optic axis of the lens $(\mu=0)$ and $7 \mathrm{~B}$ shows the fringes when $\mu=1 / 2$ unit $(=2 \mathrm{~cm})$. Eight reference points were used for this test. These were located at distances of $+2, \pm 6$, \pm 10 , and $\pm i 4 \mathrm{~cm}$ from the optical axis. The unit separation is $4 \mathrm{~cm}$. Therefore the absolute values for $\nu$ and $\sigma$ are $0.5,1.5,2.5$, and 3.5 units. Table 1 shows the observed orders of interference for the two sets of fringes at the pairs of reference points indicated by the $\nu$ and $\sigma$ columns. The straightness of the fringes in figure $7 \mathrm{~A}$ shows the wave to be symmetrical about the dividing plane. When straight fringes are obtained for several azimuthal positions of the lens (by rotating it relative to the prism about its optical axis) the test indicates the lens to be a figure of revolution. This means that $\delta_{\nu}=\delta_{\sigma}$ and eqs (4), (5), and (6) are not required. The solution for the $\delta$ 's can, therefore, be obtained by using only three observation equations and two equations of condition. The equations of condition may be based on either one of the three criteria: i.e., method of averages, method of coincidence, or method of least squares.

We shall arbitrarily choose to use the method of averages and select the reference circle by requiring that

$$
\left.\begin{array}{l}
\delta_{0.5}+\delta_{1.5}=0 \\
\delta_{2.5}+\delta_{3.5}=0 .
\end{array}\right\}
$$

The equations of observation are obtained by substituting corresponding values for $\nu, \sigma, \mu$, and $N_{\nu \sigma}$, shown in table 1 , in the columns under $(\mu=0.5)$, into eq (3). These are:

$$
\left.\begin{array}{lll}
\delta_{1.5}=\delta_{0.5}+0.55 & -1 & \epsilon_{0.5} \\
\delta_{2.5}=\delta_{1.5}+0.20 & -2 & \epsilon_{0.5} \\
\delta_{3.5}=\delta_{2.5}-0.35 & -3 & \epsilon_{0.5 .}
\end{array}\right\}
$$

Equations (22) and (23) contain 5 unknowns, $4 \delta$ 's and $\epsilon_{0.5}$. The simultaneous solution of these equations for the $\delta$ 's yields the deviations of the wave front from a sphere. These values are -0.23 . $+0.23,+0.28$, and -0.28 , respectively, for $\delta_{0.5}$, $\delta_{1.5}, \delta_{2.5}$, and $\delta_{3.5}$. Since the light passes through the lens twice in this test these deviations are twice the values that would have been obtained for one

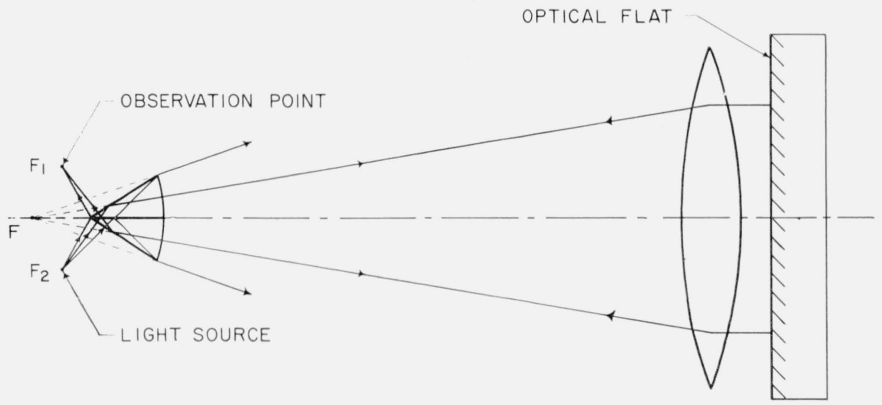

Figure 6. Optical arrangement for testing a lens with one conjugate at infinity. 


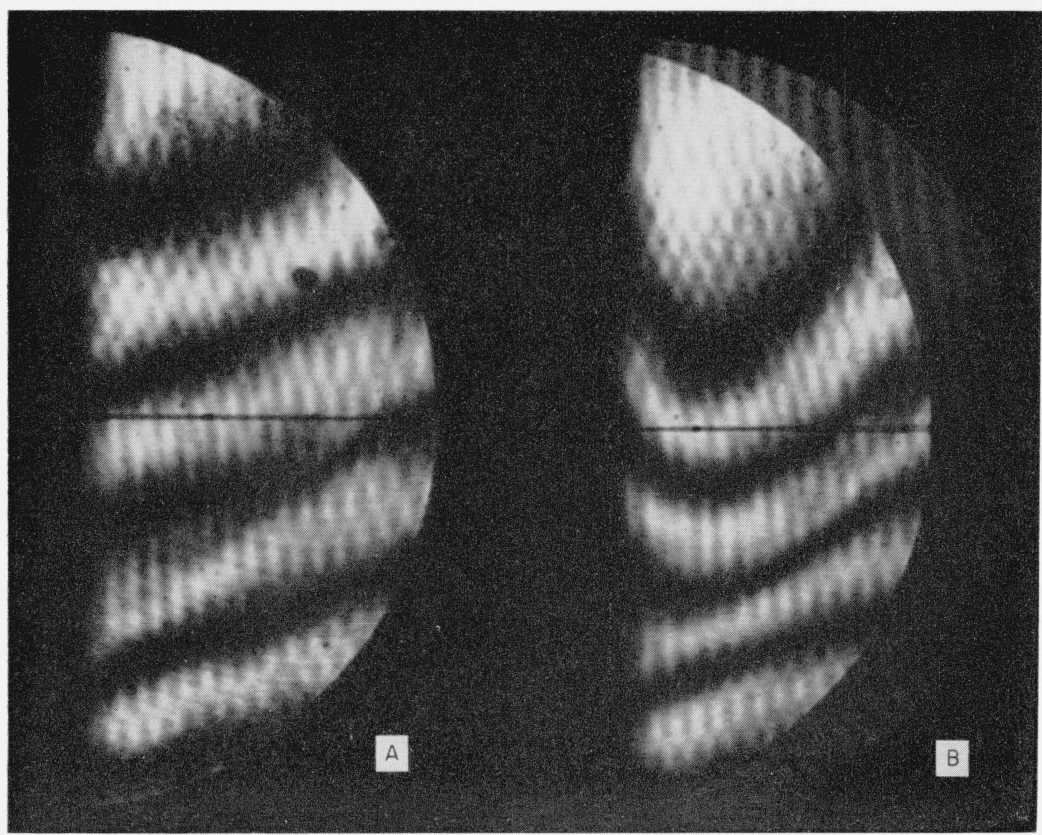

Figure 7. Photographs of fringes with 12inch aperture, $f / 15$ astronomical objective when (A) $\mu=0$, and (B) $\mu=0.5$.

TABLE 1. Data from figure $\%$ for analysis of a 12-inch aperture astronomical objective

\begin{tabular}{|c|c|c|c|c|c|}
\hline \multicolumn{3}{|c|}{$\mu=0$} & \multicolumn{3}{|c|}{$\mu=0.5$} \\
\hline$\nu$ & $\sigma$ & $N_{\nu \sigma}$ & $\nu$ & $\sigma$ & $N_{\nu \sigma}$ \\
\hline $\begin{array}{l}0.5 \\
1.5 \\
2.5 \\
3.5\end{array}$ & $\begin{array}{l}-0.5 \\
-1.5 \\
-2.5 \\
-3.5\end{array}$ & $\begin{array}{l}-0.10 \\
-0.45 \\
-0.70 \\
-1.00\end{array}$ & $\begin{array}{l}0.5 \\
1.5 \\
2.5 \\
3.5\end{array}$ & $\begin{array}{r}0.5 \\
-0.5 \\
-1.5 \\
-2.5\end{array}$ & $\begin{array}{r}0.00 \\
0.55 \\
0.20 \\
-0.35\end{array}$ \\
\hline
\end{tabular}

transmission through the lens - as for light from a celestial star. The deviations for one transmission are represented graphically in figure 8. The curve indicates that when this objective is used for astronomical work the wave front departs from a perfect sphere by approximately one-third of a wavelength. The light used for this test was the green line of the mercury spectrum $(\lambda=0.546$ microns).

If eq (3) is used in the testing of a parabolic mirror the computed deviations will be the deviations of the mirror from a perfect sphere. Usually, when testing a parabolic mirror one wishes to know how much the mirror differs from a perfect paraboloid. If the mirror is ellipsoidal one wishes to know both the deviations and the eccentricity. The eccentricity, $e$, enters as an additional parameter (eq (30) of appendix A).

The simultaneous evaluation of the deviations and the eccentricity is obtained with the method of coincidence by requiring the curve to pass through 4 points instead of 3 as was the case (eq (10)) for a

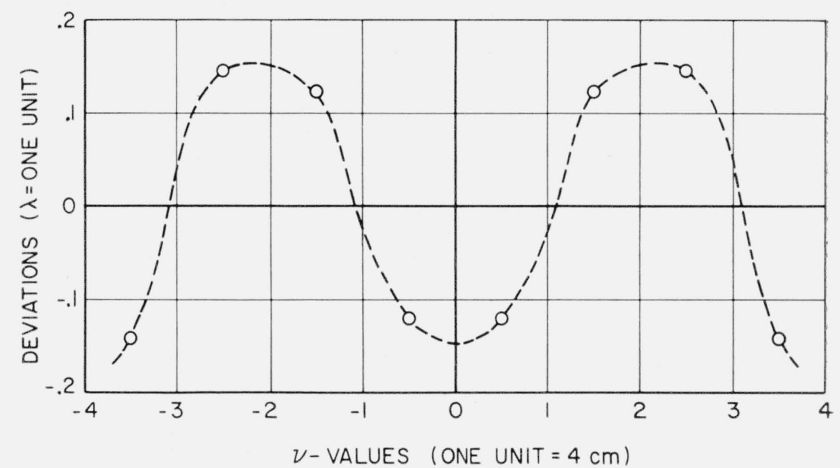

FIGURE 8. Graphical representation of wave-front-shape computed from the photographs of figure $\%$.

3-parameter reference curve. If the method of averages is to be used the reference points are grouped into 4 groups yielding 4 equations of condition instead of the 3 shown in eq (11). Similarly, with the method of least squares an additional equation $(\partial \phi / \partial e=0)$ is to be added to the three equations under eq (12). If the surface is known, or can be assumed, to be a figure of revolution about its axis, the number of condition equations is reduced by 1 , as for the circular reference curve.

Figure 9 is a photograph of fringes taken with a 12-inch aperture, 84-inch focal length, mirror that was claimed to be a nearly perfect paraboloid. Figure 10 shows the optical arrangement used for making this photograph. The computed shape of this mirror indicated that it conformed more closely to an ellipsoid than to a paraboloid. The general equation for an ellipse (described in appendix $\mathbf{A}$, eq (31)) was 


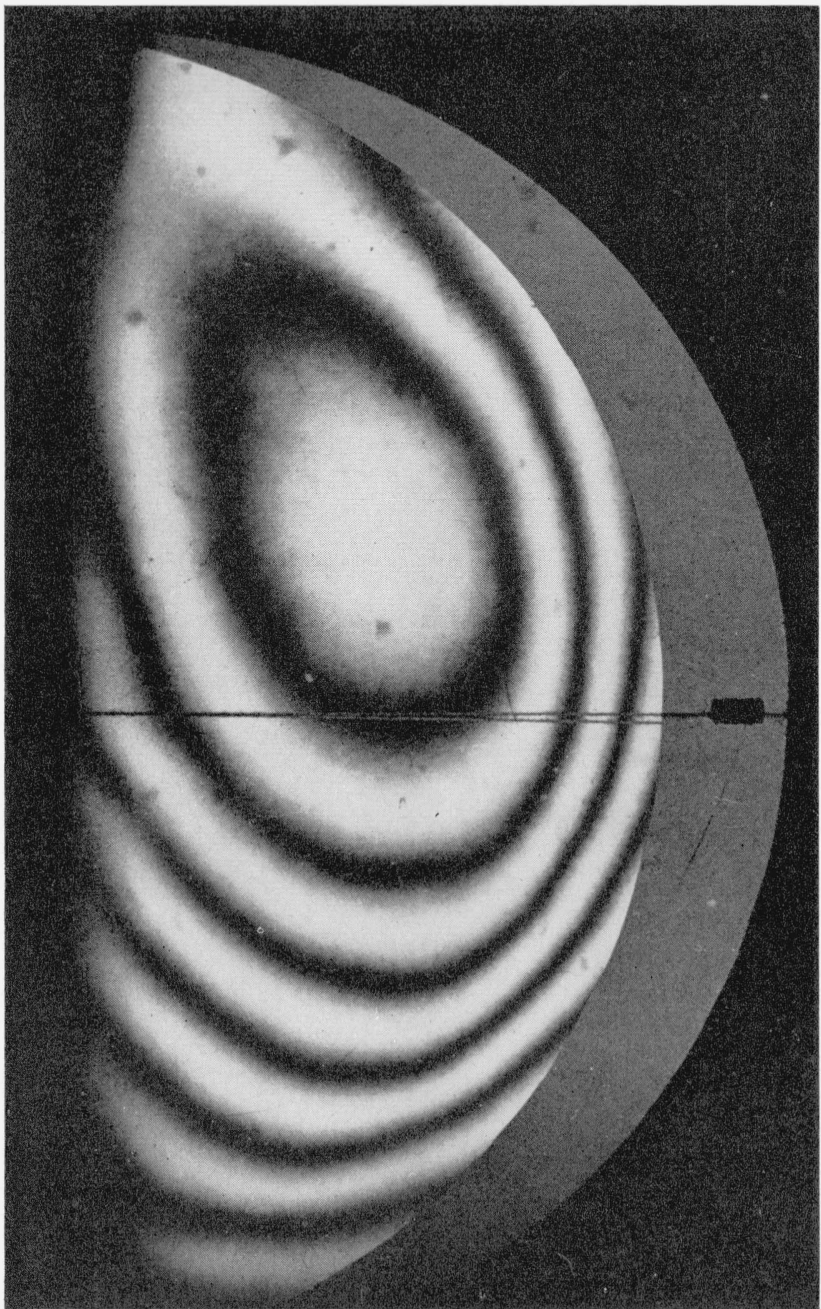

Figure 9. Photograph of fringes taken with a 12-inch aperture f/y parabolic mirror.

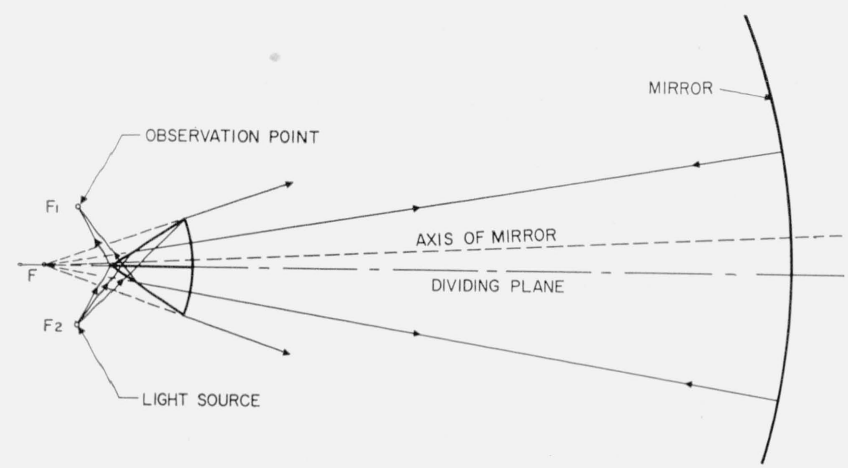

FIgure 10. Optical arrangement for testing concave mirrors at their centers of curvature.

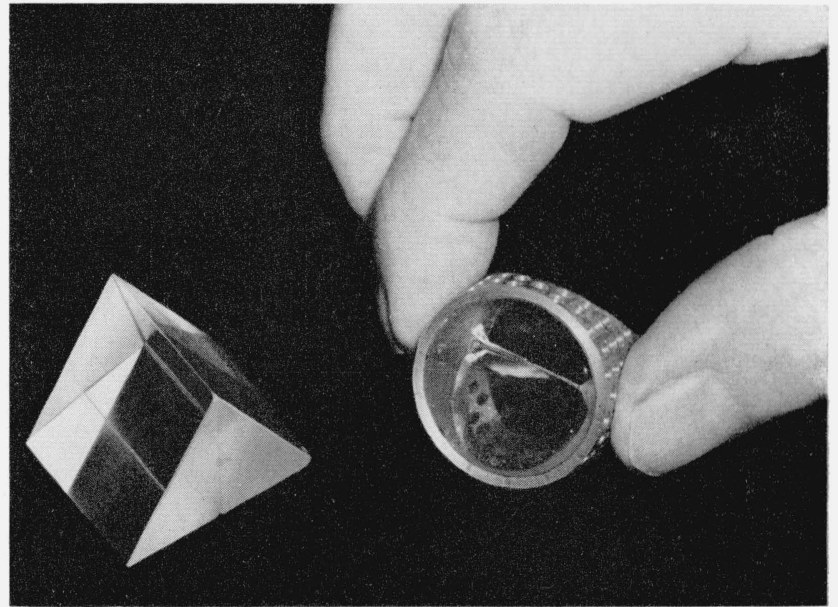

Figure 11. Photograph of two Koesters double-image prisms, one mounted in a cell and the other untrimmed.

then tried and the computed value for the eccentricity corresponded to an ellipse whose foci are separated by 89 meters. It was subsequently learned that the final test on this "Nearly Perfect Parabolic Mirror" was made with a Foucoult knife edge test, with the source located 90 meters (assumed to be practically at infinity) from the mirror and the knife edge at the point of convergence or image of the source. This test, therefore, confirms the description of the mirror as being a "nearly perfect parabola" but even more nearly a perfect ellipsoid.

Figure 11 shows photographs of the prism, before and after it is fitted into a cell. This prism has been made in sizes from approximately 6 inches down to a fraction of an inch. The one shown here has a one inch aperture and is considered a most favorable size for many applications.

\subsection{Summary}

The interferometer described here for testing wave fronts is believed to be easier to operate than any previous interferometer yet developed for this purpose. Its operation is simple. Unskilled personnel can be trained to operate it after a short period of training.

This interferometer yields results that are absolute. It does not require a standard of reference. The absolute evaluation of wave-front shapes, however, depends upon a unique method of analyzing the data. The analysis involves the development of a set of linear simultaneous equations. This requires some technical background knowledge. After the equations for a given assembly are developed, unskilled personnel can compute wave-front shapes by substituting observed orders of interference into the equations and solving for the deviations from the chosen reference curve. The chosen reference curve will usually be a circle but more complicated curves may be used. 


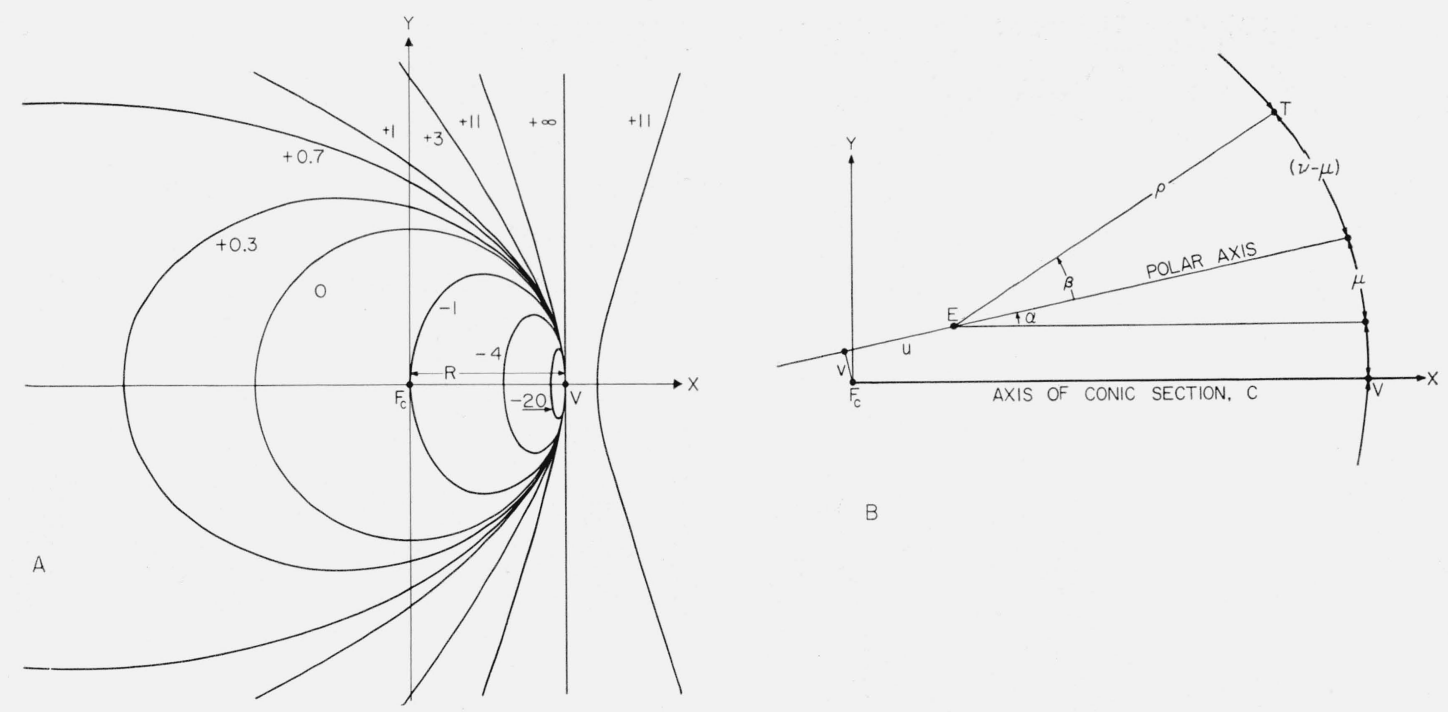

Figure 12. A. The family of curves representing the conic section for various indicated values of $e^{2}(e=e c c e n t r i c i t y)$. B. Illustration for describing the transformation from rectangular to polar coordinates.

\section{Appendix A. Optical Path Difference Between Any Two Points on a Conic Sec- tion to Any Point in the Neighborhood of the Center of Curvature of the Section at Its Vertex}

The equation for the conic section in rectangular coordinates, with the origin at $F_{c}$, (a distance $R$ to the left of the curve, see fig. $12 \mathrm{~A}$ ), and the $x$-axis coinciding with the axis of the conic, is given by the 2 parameter equation

$$
y^{2}+\left(1-e^{2}\right)(x-R)^{2}+2 R(x-R)=0 .
$$

The family of curves, shown in figure $12 \mathrm{~A}$ represents this equation for the various indicated values of $e^{2}$. The parameter $R$ is the radius of curvature of the curves at the vertex $V$ (except for $e^{2}= \pm \infty$ ), and $e$ is the eccentricity.

If we shift to polar coordinates $(\rho, \beta)$ with the polar axis making angle $\alpha$ with the axis of the conic (see fig. $12 \mathrm{~B}$ ) and the pole located at point $E$, which is displaced from $F_{c}$ by a small amount $u$ parallel to the polar axis and a small distance $v$ perpendicular to it, we may obtain the polar equation for the conic by means of the transformation equations,

$$
\left.\begin{array}{l}
y=u \sin \alpha+v \cos \alpha+\rho \sin (\alpha+\beta) \\
x=u \cos \alpha-v \sin \alpha+\rho \cos (\alpha+\beta) .
\end{array}\right\}
$$

The quantities $u$ and $v$ will never exceed the separation of points $E$ and $F_{c}$, which is always quite small relative to $\rho$, because these two points can always be superimposed to a high degree of accuracy.

On eliminating $x$ and $y$ from eqs (24) and (25); solving for $\rho$; applying binomial expansions to eliminate radicals and negative power têrms; expanding: $\sin (\alpha+\beta)$ and $\cos (\alpha+\beta)$ in powers of $(\alpha+\beta)$ [5]; and dropping all power terms in $u$ and $v$ higher than unity (because these terms are always insignificantly small), we obtain

$$
\rho=f_{1}(\alpha, \beta)-u f_{2}(\alpha, \beta)-v f_{3}(\alpha, \beta),
$$

where

$$
\left.\begin{array}{l}
f_{1}(\alpha, \beta)=R+1 / 8 \operatorname{Re}^{2}(\alpha+\beta)^{4}-1 / 48 \operatorname{Re}^{2}\left(1+3 e^{2}\right)(\alpha+\beta)^{6}+\ldots \\
f_{2}(\alpha, \beta)=\cos \beta+1 / 2 e^{2} \beta(\alpha+\beta)^{4}+\ldots \\
f_{3}(\alpha, \beta)=\sin \beta-1 / 2 e^{2}(\alpha+\beta)^{4}+\ldots
\end{array}\right\}
$$



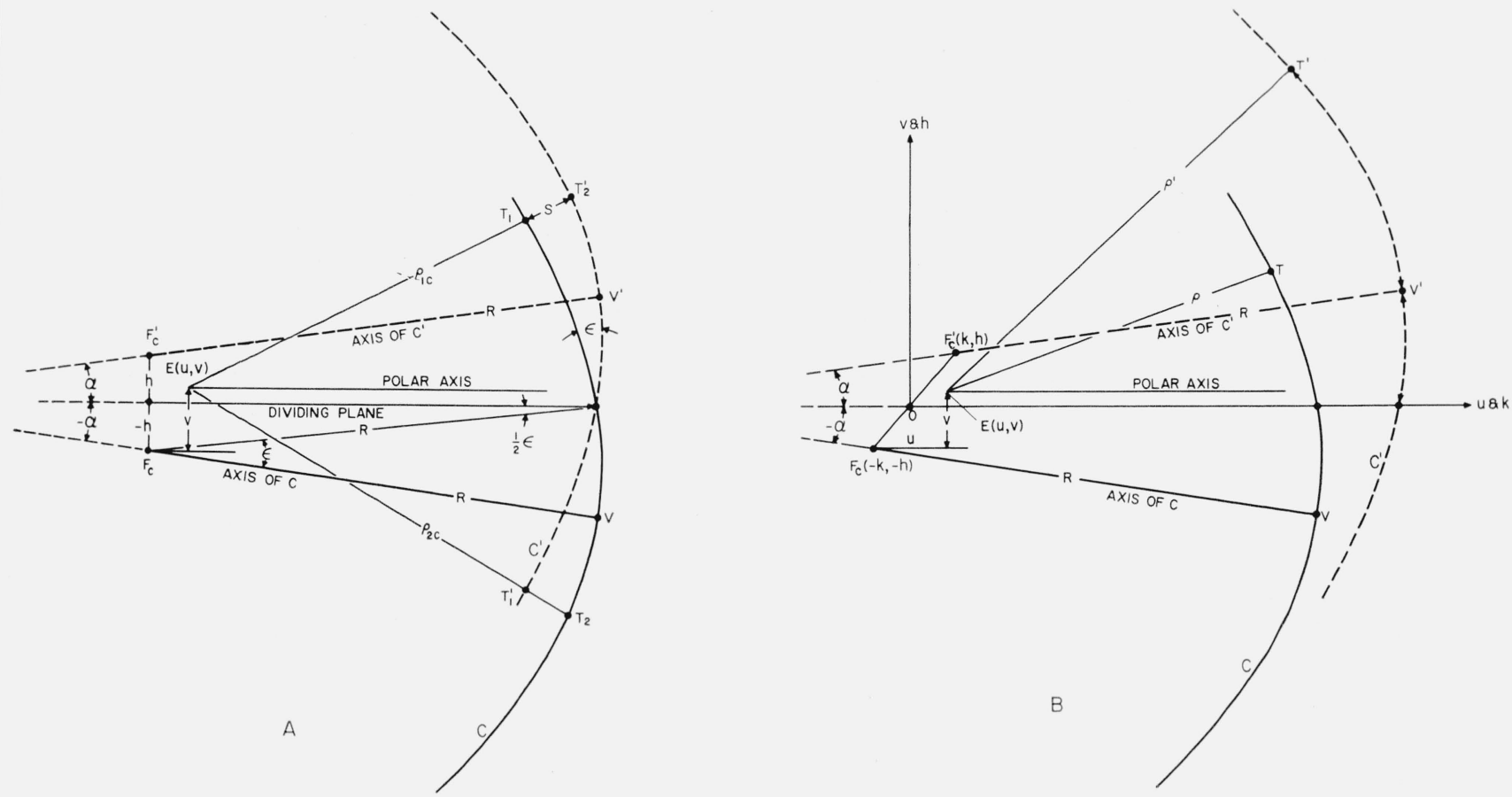

FIGURE 13. A and B. Illustration of the relative positions of two images of a wave front with the WRI and WSI, respectively.

The term $f_{1}(\alpha, \beta)$ is the value of $\rho$ when the pole is located at $F_{c}$. When testing optics of large apertureto-focal-length ratios the quantity $f_{1}(\alpha, \beta)$ must include higher order terms of the series than for smaller $f$-values. It will be found that, in some cases, the $f_{1}(\alpha, \beta)$-series converges too slowly for practical application and that the rigorous, unexpanded value

$$
f_{1}(\alpha, \beta) \equiv \frac{R\left[\sqrt{1+e^{2} \sin ^{2}(\alpha+\beta)}-e^{2} \cos (\alpha+\beta)\right]}{\left[1-e^{2} \cos ^{2}(\alpha+\beta)\right]}
$$

is more practical. For this treatment we shall limit the angles to values comparable to those obtained with an $f / 8$ system. This limitation makes the last terms, indicated in each of the functions $f_{1}(\alpha, \beta)$ $f_{2}(\alpha, \beta)$ and $f_{3}(\alpha, \beta)$ of eq (26) negligible so that eq (26) becomes

$$
\rho=R+1 / 8 R e^{2}(\alpha+\beta)^{4}-u \cos \beta-v \sin \beta .
$$

\subsection{Equations for the WRI}

When the conic is used as the reference surface in a wave-front-reversing interferometer, the optical path difference, $S$ (fig. 13A), is the difference between two radius vectors, $\rho_{1 c}$ and $\rho_{2 c}$, forming angles with the dividing plane, which are equal in magnitude but opposite in sign.

The two curves, $C$ and $C^{\prime}$, shown in figure $13 \mathrm{~A}$ are images of the conic section. These two images are inverted by the interferometer, with respect to each other, about the dividing plane which is parallel to the polar axis. The two points $T_{1}$ and $T_{1}^{\prime}$ are images of a common point on the original curve. Similarly $T_{2}$ and $T_{2}^{\prime}$ are images of a common point; but $T_{1}$ and $T_{2}$ are images of different points that are at equal angular distances from the dividing plane. The radii vectors to $T_{1}$ and $T_{2}$ are $\rho_{1 c}$ and $\rho_{2 c}$, respectively.

If we define the perpendicular displacement of $F_{c}$ from the dividing plane as $-h$, the corresponding displacement for $F_{c}^{\prime}$ is $+h$. The component displacements of $E$ from $F_{c}$, as defined for figure 12 , is $u$ and $v$. The corresponding displacements of $E$ from $F_{c}^{\prime}$ is $u$ and $(v-2 h)$. The value of $\rho_{1 c}$ is, therefore, obtained by replacing $\rho$ in eq $(28)$ by $\rho_{1 c}$. Similarly, since the $\operatorname{sign} \alpha$ is reversed in the image, the value for $\rho_{2 c}$ is obtained by replacing $\rho, \alpha$, and $v$ by $\rho_{2 c},-\alpha$, and $(v-2 h)$, respectively. On making these substitutions, we obtain

$$
\rho_{2 c}-\rho_{1 c} \equiv S=2 h \sin \beta-R e^{2} \alpha \beta\left(\alpha^{2}+\beta^{2}\right) .
$$


It is convenient to use the measured distances $(\nu-\mu)$, and $\mu$ instead of the angles $\beta$ and $\alpha$. Also, from figure $13 \mathrm{~A}$, it is obvious that $2 h=\epsilon R$, since $h$ is quite small relative to $R$. On substituting for $2 h, \alpha$, $\beta$ ( $=\sin \beta$, approximately), their respective equals, $\epsilon R, \mu R^{-1}$, and $(\nu-\mu) R^{-1}$, the value of $S$ becomes

$$
S=\epsilon(\nu-\mu)-e^{2} R^{-3} \mu(\nu-\mu)\left[\mu^{2}+(\nu-\mu)^{2}\right]
$$

which becomes for a paraboloid $(e=1)$,

$$
S=\epsilon(\nu-\mu)-R^{-3} \mu(\nu-\mu)\left[\mu^{2}+(\nu-\mu)^{2}\right]
$$

and for a sphere $(e=0)$,

$$
S=\epsilon(\nu-\mu) .
$$

Equation (32) is the same as eq (1). The use of a conic section instead of a circle for the reference curve, in the derivation of eq (3), leads to the introduction of the additional unknown, $e$, into eq (3) so that an additional condition equation becomes necessary for a unique solution, thus permitting the incidental evaluation of $e$. Since four conditions are required to define the chosen conicoid, four condition equations are needed. These may be: The assignment of arbitrary values (usually zeros) to any four of the $\delta$ 's (if the method of coincidence is used); the grouping of the reference points into four groups and requiring the algebraic sum of the $\delta$ 's in each group to be zero (if the method of averages is used); or, the addition of the condition equation, $\frac{\partial \varphi}{\partial e}=0$, to eq (12) (if the method of least squares is used).

\subsection{Application to the WSI}

When the conic is used as a reference surface in a wave-front-shearing interferometer, the optical path difference, $S$, is $\left(\rho^{\prime}-\rho\right)$, where $\rho$ and $\rho^{\prime}$ are the radius vectors from the point $E$ to points $T$ and $T^{\prime}$ (see fig. 13B) on curves $C$ and $C^{\prime}$, respectively. Points $T$ and $T^{\prime}$ are images of a common point on the conic section. Curves $C$ and $C^{\prime}$ are sheared images of the conic section. The angle of shear is $-\alpha$ for $C$ and $\alpha$ for $C^{\prime}$. Therefore, the polar axis makes angles $\alpha$ and $-\alpha$ with the axis of $C$ and $C^{\prime}$, respectively.

The component displacements of $E$ from $F_{c}$ is the same in figure $13 B$ as in figure $12 B$. The value for $\rho$ is, therefore, given by eq (28). The component displacements of $E$ from $F^{\prime}$ is $(u-2 k)$ and $(v-2 h)$. Consequently, the value for $\rho^{\prime}$ is obtained from eq (28) by replacing $\rho, \alpha, u$, and $v$ by $\rho^{\prime},-\alpha,(u-2 k)$ and $(v-2 h)$, respectively. On making these substitutions and taking differences we obtain

$$
\rho^{\prime}-\rho \equiv S=2 h \sin \beta+2 k \cos \beta-R e^{2} \alpha \beta\left(\alpha^{2}+\beta^{2}\right) .
$$

It will be noted that $k$ does not appear in eq (29) (relating to WRI) but does appear in (33) (relating to WSI). The term $2 k \cos \beta$ is approximately constant when $\beta$ is limited to small values (as when testing an $f / 8$ system) but becomes significant for large values of $\beta$ (as when testing an $f / 1$ system), unless $k$ is sufficiently small. If the WSI is adjusted to place the zero order of interference at the center, the magnitude of $k$ is insignificant and the values of $S$ for the two interferometers are identical. On substituting for $2 h, \alpha$, and $\beta$ their respective equals, as given in section 3.1, above ( $\cos \beta=1$, approximately), eq (33) becomes

$$
S=\epsilon(\nu-\mu)+2 k-e^{2} R^{-3} \mu(\nu-\mu)\left[\mu^{2}+(\nu-\mu)^{2}\right]
$$

which becomes for a paraboloid $(e=1)$,

$$
S=\epsilon(\nu-\mu)+2 k-R^{-3} \mu(\nu-\mu)\left[\mu^{2}+(\nu-\mu)^{2}\right]
$$

and for a sphere $(e=0)$,

$$
S=\epsilon(\nu-\mu)+2 k .
$$

It is inconvenient and unnecessary to reduce $k$ to an insignificant magnitude. It can be combined with other constants, as described in reference [2]. In fact, the constant $k$ is a part of the constant $r$ of reference [2]. Unfortunately, the sign convention for measuring $\mu$ and $\nu$ is different in this paper (part 2 ) from that of part 1 [2]. The constant, $r$, computed from eq (3) of reference [2] in combination with eq (36) above, becomes $r=Q_{r}+\epsilon \mu-2 k$. Since $r$ is a constant and serves as a parameter only, its form does not affect the problem.

\section{References}

[1] J. B. Saunders, Construction of a Koesters double-image prism, J. Research NBS 58, 21 (1957) RP2729.

[2] J. B. Saunders, Measurement of wave fronts without a reference standard: Part I. The wave-front-shearing interferometer; J. Research NBS 65B, 239 (OctoberDecember 1961).

[3] Pol Mollet, Optics in metrology, the wavefront reversing interferometer, p. 227 (The Pergamon Press, 1960).

[4] J. B. Scarborough, Numerical mathematical analysis, p. 357 (The Johns Hopkins Press, Baltimore, Md., 1930).

[5] W. A. Granville, Elements of the differential and integral calculus, p. 228; Ginn and Co. (or any other textbook on differential and integral calculus).

[6] J. B. Saunders, Parallel testing interferometer, J. Research NBS 61, 491 (1957) RP 2917. 


\title{
Publications of the National Bureau of Standards*
}

\author{
(Including Papers in Outside Journals)
}

\section{Selected Abstracts}

Fractional factorial designs for experiments with factors at two and three levels, W. S. Connor and S. Young, NBS Applied Math. Series 58 (1961) 40 cents.

This publication contains fractional factorial designs for use in experiments which investigate $m$ factors at 2 levels and $n$ factors at three levels. The grand mean, all main effects, and all two-factor interaction effects are estimated. All higher-order interactions are assumed negligible and their absence allows estimation of the error variance. A design has been constructed for each of the 39 pairs $(m, n)$ included from $m+n=5$ through $m+n=10,(m, n \neq 0)$.

An evaluation of Kacser's second order Born approximation to the bremsstrahlung differential cross section, G. S. Ofelt, NBS Tech. Note 81 (PB161582) (1961) 75 cents.

The second order term, as derived by C. Kacser [Proc. Roy. Soc. A253 (1959)], of the Born approximation series for the bremsstrahlung cross section differential with respect to photon energy, photon direction, and final electron direction averaged over initial and summed over final polarization states has been put in a form that admits to numerical evaluation for the coplanar case. The results are valid for relativistic as well as non-relativistic incident electrons.

Completely general (noncoplanar) expressions are included for the first order (Bethe-Heitler) and second order terms. However, the latter has not been given in a form admissible to numerical evaluation.

Tabulated values are given for the case of the incident electron kinetic energy $=500 \mathrm{kev}$, photon energy $=450 \mathrm{kev}$, and photon direction $20^{\circ}$ from the incident electron direction. For this case the second order term increases the differential cross section by about 50 percent for $\mathrm{Al}$ and about 200 percent for $\mathrm{Au}$ for the various angles of electron emission. A rough approximation to the integrated (over final electron direction) cross section is included and a comparison is made with experimental values. The inclusion of the second order term brings the theoretical cross section closer to the experimental values.

\section{An approximate full wave solution for low frequency electro-} magnetic waves in an unbounded magneto-ionic medium, W. C. Hoffman, J. Research NBS 66D (Radio Prop.) No. 1, 107 (Jan.-Feb. 1962), 70 cents.

Maxwell's equations in an anisotropic inhomogeneous medium are transformed by means of the Stratton-Chu formula into a vector integral equation which couples the various electric field components. In case the hypotheses of far-zone field and low-frequency electromagnetic waves apply, this vector integral equation can be approximated by a system of uncoupled scalar integral equations. This implies an approximate equivalence between the original vector integral equation and a system of modified scalar inhomogeneous Helmholtz equations. The conditions under which the system of uncoupled scalar integral equations can be solved by Neumann series are discussed, and the first three terms of the Neumann series are given explicitly.

Current on and input impedence of a cylindrical antenna, Y. M. Chen and J. B. Keller, J. Research NBS 66D (Radio Prop.) No. 1, 15 (Jan.-Feb. 1962) 70 cents.

The current on a finite antenna is expressed as the sum of a current emanating from the gap and two currents reflected from the ends. These currents are determined for a perfectly conducting hollow pipe of circular cross section. It is excited by an electric field parallel to the axis applied across a gap of finite width which encircles the antennas. They are also determined for a thin antenna of any cross section. From the results the current on and input admittance of the corresponding finite antennas are determined. It is shown that the thin antenna theory yields an incorrect result for the admittance because it ignores a boundary layer effect near the gap.

On the propagation of VLF and ELF radio waves when the ionosphere is not sharply bounded, J. R. Wait, J. Research NBS 66D (Radio Prop.) No. 1, 53 (Jan.-Feb. 1962) 70 cents. Employing an idea of Brekhovskikh, an expression for the reflection coefficient of a continuously stratified ionized medium is derived. The result is in the form of a series whose first term is a Fresnel-type coefficient and succeeding terms account for the finite thickness of the transition layer. This result is then fitted into previously developed theory for propagation between a spherical earth and a concentric ionosphere.

On the theory of wave propagation through a concentrically stratified troposphere with a smooth profile. Part II. Expansions of the rigorous solution, $\mathrm{H}$. Bremmer, $J$. Research NBS 66D (Radio Prop.) No. 1, 31 (Jan.-Feb. 1962) 70 cents.

This part concerns the height-gain differential equation in order to obtain a series for the complete solution which starts with the extended W. K. B. approximation discussed in part I. The coefficients of this equation depend for each mode amongst other things on the parameters $\boldsymbol{\Lambda}_{\mathrm{j}}$ fixing the refractive-index profile. However, the explicit dependence on these parameters can only be given in terms of expansions with respect to $\left(k_{0} a\right)^{-2 / 3}\left(k_{0} a=\right.$ circumference of the earth divided by the wavelength). In turn these expansions are derived with the aid of other ones for the complex turning point connected with the height-gain differential equation. The final expansion for the solution of the differential equation is substituted in the boundary condition at the earth's surface. This leads to corresponding expansions, with respect to $\left(k_{0} a\right)^{-2 / 3}$ of the quantity $u \lambda(a)$ and next of the eigenvalues $l$ themselves.

Reflection and transmission of radio waves at a continuously stratified plasma with arbitrary magnetic induction, J. R. Johler and J. D. Harper, Jr., J. Research NBS 66D (Radio Prop.) No. 1, 81 (Jan.-Feb. 1962) 70 cents.

A flexible theoretical model plasma which can be deformed to fit most measured electron-ion-altitude profiles is employed together with available geophysical data on the ionosphere to evaluate reflections and transmissions during quiescent and disturbed propagation conditions. The reflections and transmissions in the ionosphere are determined rigorously with the aid of the classical magneto-ionic theory. The complex indexes of refraction of the medium are deduced, and a coupling in the plasma between ordinary and extraordinary, upgoing and downgoing modes of propagation is investigated. The corresponding reflection and transmission coefficients are then calculated, and certain phenomena which can be expected as a result of the action of a solar disturbance on the reflection process are predicted.

The disturbance of solar origin, investigated as an application of developed techniques, influences the reflected and transmitted LF waves in the lower ionosphere in a complicated manner. However, the high absorption phenomena exhibited by high frequencies does not seem to exist for the plasma profiles investigated with the classical magneto-ionic theory. The electron collision frequencies of the classical magneticionic theory are modified to introduce a collision frequency proportional to the electron energy, and the changes necessary in the formulation of the classical theory as a result of such a consideration are presented. 
On the diffraction of spherical radio waves by a finitely conducting spherical earth, L. C. Walters and J. R. Johler, $J$. Research NBS 66D (Radio Prop.) No. 1, 101 (Jan.-Feb. 1962) 70 cents.

The theory for the diffraction of spherical electromagnetic waves by a finitely conducting spherical earth was developed from Maxwell's equations by Watson [1918] and the intricate computation details were later worked out by van der Pol and Bremmer [1936] as the now classical series of residues. Two aspects of this computation present considerable difficulty, especially at low frequencies: (1) the calculation of the height-gain factor which takes account of an elevated transmitter and/or receiver, (2) the evaluation of the special roots $\tau=\tau_{s}$ of Riccati's differential equation, $d \delta / d \tau$ $-2 \delta^{2} \tau+1=0$, near the circle of convergence, $\left|\delta^{2} \tau\right|=1 / 2$.

These analytic difficulties are avoided with the aid of modern analysis techniques applied to a large scale electronic computer. Hankel functions of the first and second kind of order one-third and two-thirds are calculated by numerical integral methods and then used with iteration to solve Riccati's differential equation. The amplitude and phase of the spherical radio wave diffracted in the vicinity of the earth with various altitudes above the surface of the earth of both the transmitter and the receiver, are then calculated by a summation of the series of residues.

The electromagnetic fields of horizontal dipole in the presence of a conducting half space, J. R. Wait, Can. J. Phys. 39, No. \%, 101\%-1028 (July 1961).

The problem considered is a horizontal electric dipole which is located above or below the plane surface of a conducting half-space. Expressions for the fields are obtained using three different approaches. The formulas developed are quite simple and, taken together, the whole range of distances from the far-zone to near-zone is adequately covered.

Simple calculus for all-dielectric interference filters, K. D. Mielenz, J. Opt. Soc. Am. 50, No. 10, 1014 (Oct. 1960).

Chebychev polynomials are introduced into the matrix theory of first order all-dielectric interference filters consisting of $2 m$ or $(2 m+1)$ alternating high and low index films of equal optical thickness $\beta$ on each side of the low index spacer of thickness $2 \beta$. For the amplitude and energy transmitted by such filters, simple closed expressions are derived that will render numerical results with greater facility than any other ealculus known.

Bounds for determinants with positive diagonals, $E$. $V$. Haynsworth, Trans. Am. Math. Soc. 96, 395-399 (1960).

A new bound is given for determinants with "dominant" diagonals, that is those for which $a_{\mathrm{ii}} \geq \sum_{j=1}^{n} a_{\mathrm{ij}}(i=1, \ldots, n)$, and another is given for determinants whose diagonals are positive and satisfy the hypotheses of a theorem by $\mathrm{A}$. Ostroski.

The classical field theories, C. Truesdell and R. Toupin, Handb. der Physik, Springer, Berlin, III, No. 1, 226-793 (1960).

This paper presents an organized account of everything known concerning general continuous media.

Resonance characteristics of a corrugated cylinder excited by a magnetic dipole, J. R. Wait and A. M. Conda, IRE Trans. Ant. Prop. AP-9, 330-33S (July 1961).

Radiation from an axial magnetic current element in the presence of a corrugated cylinder is considered. It is indicated that the power radiated in a given mode depends on the surface reactance, the circumference of the cylinder and elevation angle. For certain values of the parameters particular modes are strongly excited, corresponding to a resonance condition of the circumferential (or spiral) surface waves.

The calculation of energy levels of nearly symmetric rotors, S. C. Wait, Jr., J. Mol. Spectroscopy 6, 276 (1960).

A recursion relation is developed for expressing the inverse of a power series by another power series. The derived relation is exact for any desired degree of approximation.
An example of this recusion formula is given for use with an extended perturbation treatment of the nearly symmetric rotor.

Theory of vibrational relaxation in liquids, R. Zwanzig, J. Chem. Phys. 34, 1931-1935 (1961).

A new formulation of the theory of vibrational relaxation, based on Zener's semiclassical approximation, is presented here. The relaxation rate is shown to be proportional to the spectral density of the force exerted on the oscillator by its environment. The isolated binary collision theory is derived, but only with the condition that the collision frequency is much smaller than the oscillator frequency. This requirement is not satisfied in a liquid; we conclude that Litovitz's application of the isolated binary collision theory to liquids is not justified. A possible relation between vibrational relaxation and the self-diffusion coefficient in a liquid is discussed.

Density fluctuations and heat conduction in a pure liquid, R. E. Nettleton, Phys. Fluids 4, No. 1, 74 (Jan. 1961).

Two components of the heat flux in a pure liquid are identified-one carried by sound waves and the other by diffusing molecules. Coupled relaxation equations are obtained for these components, both depending on the density gradient $\nabla \rho$, and this dependence is interpreted thermodynamically as a further coupling of these equations with the equation determining $(\partial / \partial t)(\nabla \rho)$. The latter can be immediately written down with the aid of Onsager's theorem and an inertial principle developed in a previous paper. The thermodynamic interpretation of these rate equations also leads to an explicit expression for the coefficient of $(\nabla \rho)^{2}$ in a Taylor expansion, about equilibrium, of the Helmholtz free energy. This result is compared with similar free energy terms assumed to exist by other authors.

Non-reciprocity and time-reversal in microwave circuits, H. A. Fowler, Academie royale de Belgique (Classe Des Sciences) V-46, No. 11, 963-97\% (Dec. 1960).

The relation between reciprocity of the microwave scattering matrix and a "local" time-reversal symmetry of the scattering process is discussed, under the unitary condition. The theory of reciprocal junctions is extended to include nonreciprocal ferrite devices: the $\gamma$-circulator of Chait and Curry is analyzed in accordance with the point-group method.

Tchebycheff approximations by $a b^{x}+c$, J. R. Rice, J. Soc. Ind. Appl. Math. 8, No. 4, 691-702, (1960).

In this paper the theory of Tchebycheff approximations by $a b^{x}+c$ and $a b^{x} \cos \left(\theta_{0}+\theta x\right)+c$ is studied where $a, b, c, \theta_{\mathrm{o}}$ and $\theta$ are parameters to be studied. A complete development of the theory is made for $a b^{x}+c$. The topics considered are existence theorem, characterization of best approximations, approximations on subsets, uniqueness theorem, approximation on finite point sets, limiting relations between approximations on $[0,1]$ and finite point sets and computational procedures for finite point sets. A really surprising phenomenon of this theory is that best approximations do not necessarily exist on finite subset of $[0,1]$, although best approximations on $[0,1]$ exist for every continuous function. The results for $a b^{x} \cos \left(\theta_{0}+\theta x\right)+c$ are very incomplete and only the rudiments of a theory are established. This is due mainly to the intractibility of the transcendental equations arising in the analysis. On the other hand some useful formulas for applications are obtained.

Repulsion of energy levels in complex atomic spectra, R. E. Trees, Phys. Rev. 123, No. 4, 1293-1300 (Aug. 15, 1961).

Rosenzweig and Porter have shown a "repulsion of energy levels" in spacing distributions determined from energy levels in complex atomic spectra. The present paper extends their work by showing that these spacing distributions can be determined from calculated positions of the levels in these spectra. Since calculated data are available for spectra where the observed data are scarce or incomplete, this partially overcomes limitations imposed by statistical inaccuracy when direct use is made of the observed data. The equivalence of the two approaches is demonstrated by showing that calculated data for Ta II vield the same spacing distribution as obtained from observed data for Ta II and Re I combined. 
These are complex spectra in which a fully developed repulsion effect is present. A similar demonstration of equivalence is carried out for spectra of Ru I and Mo I, where the repulsion effect is in an intermediate state of development. The results also indicate that numbers easily evaluated from the radial parameters of the theory will indicate roughly the degree of repulsion, replacing to some extent the need for an explicit calculation of the spacing distribution.

Statistical dynamics of simple cubic lattices. Model for the study of Brownian motion II, R. J. Rubin, J. Math. Phys. 2, No. 3, 373-386 (May-June 1961).

New results concerning the statistical dynamics of a heavy particle in an $n$-dimensional $(n D)$ cubic lattice are presented. In a well-defined sense, the random motions of a heavy particle in a $1 D$ lattice and a $3 D$ lattice are accurately described by Kramers' equation for a free particle and a harmonically bound particle, respectively. A related, but not independent, result is that the velocity $v(t)$ and position $u(t)$ of a heavy particle in a $1 D$ lattice and a $3 D$ lattice constitute two dimensional stationary gaussian Markoff processes. It is definitely established that in the case of a $2 D$ lattice the station gaussian process $\left(\begin{array}{l}v(t) \\ u(t)\end{array}\right)$ is non-Markoffian. In the course of the analysis, several interesting connections between solutions of the discrete lattice equations of motion and solutions of the corresponding continuum equation of motion (the $n D$ wave equation) are uncovered.

Partial confounding in fractional replication, W. J. Youden, Technometric 3, No. 3, 353-358 (Aug. 1961).

Fractional factorial designs are based on using some fraction of the total possible number of experimental combinations of the variables. When resources permit, the experimenter may obtain both the direct effect of any variable and the effect as modified by the other variables. The latter information may be sacrificed if fewer combinations are explored. This paper presents an intermediate possibility without increasing the number of combinations. The direct and the differential effects may be identified and estimated at some sacrifice in the precision of the estimates.

Exact and approximate distributions for the Wilcoxon statistics with ties, S. Y. Lehman, J. Am. Stat. Assoc. 56, 293-298 (June 1961).

The exact distribution of the Wilcoxon statistic was calculated for five specific cases where both samples contained five observations, some of which were tied. The five specific cases were obtained from an actual experiment. The exact distributions for each of these five cases are compared with some approximations at points near 1, 5, and 10 percent. Critical values and associated probabilities have been calculated for the exact and approximate distributions.

Some geometrical theorems for abscissas and weights of gauss type, P. J. Davis and P. Rabinowitz, J. Math. Anal. Appl. 2, No. 3, 428-437 (June 1961).

Several theorems which relate Gaussian weights and abscissas asymptotically are projected and others are conjectured. Those conjectured have been established numerically.

\section{Other NBS Publications}

\section{Journal of Research 66A (Phys. and Chem.) No. 1 (Jan.-} Feb. 1962) 70 cents.

Absolute isotopic abundance of terrestrial silver. W. R. Shields, E. L. Garner, and V. H. Dibeler.

Temperature of a copper are. C. H. Corliss.

Melting process and the equilibrium melting temperature of polychlorotrifluoroethylene. J. D. Hoffman and J. J. Weeks.

Tritium-labeled compounds VIII. Confirmation of the position of the tritium in D-glucose-6-t and D-glucitol-5- $t$. L. T. Sniegoski and H. S. Isbell.

Infrared absorption spectra in the study of mutarotational equilibria of monosaccharides. R. S. Tipson and H. S. Isbell.

Preparation of high purity trimethylborane. G. S. Ross, D. Enagonio, C. A. Hewitt, and A. R. Glasgow.
Reaction of several aminopyrimidines with formaldehyde. G. L. McLeod.

Acidic dissociation constant and related thermodynamic quantities for diethanolammonium ion in water from 0 to $50{ }^{\circ}$ C. V. E. Bower, R. A. Robinson, and R. G. Bates.

Fiber structure-property relationships II: Macroscopic deformations of alkylene sulfide crosslinked polycaprolactam fibers. S. D. Bruck.

Ion transport across membranes: I. Definitions of membrane electromotive forces and of flows of electrolytic solutes. B. C. Duncan.

Journal of Research 66C (Eng. and Instr.) No. 1 (Jan.-Mar. 1962) 75 cents.

Reference tables for 40 percent iridium-60 percent rhodium versus iridium thermocouples. G. F. Blackburn and F. R. Caldwell.

A method for the self-calibration of attenuation-measuring systems. R. L. Peck.

Special shielded resistor for high-voltage d-c measurements. J. H. Parks.

Voltage ratio measurements with a transformer capacitance bridge. T. L. Zapf.

Weight calibration schemes for two knife-edge direct-reading balances. H. E. Almer, L. B. Macurdy, H. S. Peiser, and E. A. Weck.

Tunnel diode large-signal equivalent circuit study and the solutions of its nonlinear differential equations. S. B. Geller and P. A. Mantek.

A missile technique for the study of detonation waves. F. W. Ruegg and W. W. Dorsey.

Creep of cold-drawn nickel, copper, 70 percent nickel-30 percent copper, and 30 percent nickel-70 percent copper alloys. W. D. Jenkins and W. A. Willard.

Journal of Research 66D (Radio Prop.) No. 1 (Jan.-Feb. 1962) 75 cents.

A survey of the very wide band and frequency independent antennas -1945 to the present. J. D. Dyson.

Numerical investigation of the equivalent impedance of a wire grid parallel to the interface between two media. T. Larsen.

Current on and input impedance of a cylindrical antenna. Y. M. Chen and J. B. Keller. (See above abstract.)

Radar corner reflectors for linear or circular polarization. G. Latmiral and A. Sposito.

On the theory of wave propagation through a concentrically stratified troposphere with a smooth profile. H. Bremmer. (See above abstract.)

On the propagation of VLF and ELF radio waves when the ionosphere is not sharply bounded. J. R. Wait. (See above abstract.)

Fields of electric dipoles in sea water-the earth-atmosphereionosphere problem. W. L. Anderson.

Reflection of electromagnetic waves from thin ionized gaseous layers. F. H. Northover.

Reflection and transmission of radio waves at a continuously stratified plasma with arbitrary magnetic induction. J. R. Johler and J. D. Harper, Jr. (See above abstract.)

On the diffraction of spherical radio waves by a finitely conducting spherical earth. L. C. Walters and J. R. Johler. (See above abstract.)

An approximate full wave solution for low frequency electromagnetic waves in an unbounded magneto-ionic medium. W. C. Hoffman. (See above abstract.)

VHF radio propagation data for the Cedar Rapids-Sterling, Anchorage-Barrow, and Fargo-Churchill test paths, April 1951 through June 1958 . G. R. Sugar and K. W. Sullivan.

Tables of spectral-line intensities, arranged by wavelengths, W. F. Meggers, C. H. Corliss, and B. F. Scribner, NBS Mono. 32, Part II (1961) \$3.00.

International practical temperature scale of 1948, text revision of 1960, H. F. Stimson, NBS Mono. 37 (1961) 10 cents. 
Stopping powers for use with cavity chambers, NBS Handb. 79 (1961) 35 cents.

Safety rules for the installation and maintenance of electric supply and communications lines. Comprising Part 2, the definitions, and the grounding rules of the sixth edition of the national electrical safety code, NBS Handb. 81 (1961) $\$ 1.75$.

Hydraulic research in the United States, H. K. Middleton, NBS Misc. Publ. 238 (1961) \$1.25.

Quarterly radio noise data, March, April, May 1961, W. Q. Crichlow, R. T. Disney, and M. A. Jenkins, NBS Tech. Note 18-10 (PB151377-10) (1961) \$1.50.

Mean electron density variations of the quiet ionosphere, No. 5 -July 1959, J. W. Wright, L. R. Wescott, and D. J. Brown, NBS Tech. Note 40-5 (PB151399-5) (1961) $\$ 1.50$

Flux switching mechanisms in ferrite cores and their dependence on core geometry, G. W. Reimherr, NBS Tech. Note 90 (PB161591) (1961) \$1.25.

An experimental study of beta decay using the radiations from oriented nuclei, D. D. Hoppes, NBS Tech. Note 93 (PB161594) (1961) \$1.50.

Bibliography on meteoric radio wave propagation, W. Nupen, NBS Tech. Note 94 (PB161595) (1961) $\$ 2.75$.

The integrated starlight over the sky, L. R. Megill and F. E. Roach, NBS Tech. Note 106 (PB161607) (1961) \$2.00.

A compilation of the physical equilibria and related properties of the hydrogen-carbon monoxide system, D. E. Drayer and T. M. Flynn, NBS Tech. Note 108 (PB161609) (1961) \$2.25.

A compilation of the physical equilibria and related properties of the hydrogen-helium system, D. E. Drayer and T. M. Flynn, NBS Tech. Note 109 (PB161610) (1961) \$1.25.

A compilation of the physical equilibria and related properties of the hydrogen-nitrogen system, D. E. Drayer and T. M. Flynn, NBS Tech. Note 110 (PB161611) (1961) \$1.75.

Mode calculations for VLF propagation in the earth-ionosphere waveguide, K. P. Spies and J. R. Wait, NBS Tech. Note 114 (PB161615) (1961) \$1.50.

Load carrying capacity of gas-lubricated bearings with inherent orifice compensation using nigrogen and helium gas, H. Sixsmith, W. A. Wilson, and B. W. Birmingham, NBS Tech. Note 115 (PB161616) (1961) $\$ 1.00$.

Matrix elements in the forbidden beta decay of $\mathrm{Ce}^{141}, \mathrm{D} . \mathrm{D}$. Hoppes, E. Ambler, R. W. Hayward, and R. S. Kaeser, Phys. Rev. Letters 6, 115-118 (1961).

Simple adiabatic demagnetization apparatus, V. D. Arp and R. H. Kropschot, Rev. Sci. Instr. Note 32, 217-218 (Feb. 1961).

4-Nitrophenyl esters, carbonates, and bi-carbonates as leather fungicides, S. Dahl and A. M. Kaplan, J. Am. Leather Chemists Assoc. 55, 480 (Sept. 1960).

The organization of international intercomparisons of radioactivity standards, with special reference to such measurements of NBS standards, L. M. Cavallo and W. B. Mann, Proc. 1959 Symp. Metrology Radioactive, IAEA, Vienne, 117 (1960).

The physical environment as affected by radiation, D. M. Gates, Proceedings of the 50th Anniversary Celebration of the University of Michigan Biological Station (1960).

Second revision American Dental Association specifications No. 4 for dental inlay casting wax, J. W. Stanford, K. V. Weigel, and J. W. Paffenbarger, J. Am. Dental Assoc. 62, 45 (Jan. 1961).

The structure of B-type vibrational-rotational bands of an asymmetric rotor, H. C. Allen, Jr., Phil. Trans. Roy. Soc. of London, Series A, Mathematical and Physical Sciences, No. 1030, 253,335 (1961).

Measurement of contrast in the aerial image, F. W. Rosberry, Photogrammetric Eng. 155-159 (March 1961).

An interferometric instrument for the rapid measurement of small diameters, D. H. Blackburn, Rev. Sci. Instr. 32, No. 2, 137 (Feb. 1961).

Vapor-phase photolysis of formic acid, R. Gordon, Jr., and P. Ausloos, J. Chem. Phys. 65, 1033 (1961).

Binary silicate glasses in the study of alkali-aggregate reaction, R. G. Pike, D. Hubbard, and E. S. Newman, Highway Research Board Bull. No. 275, 39-44 (1960).

Complex conductivity of some plasmas and semiconductors, P. H. Fang, J. Appl. Research Soc. B (The Hague) 9, 51 (1960)
Physical measurement-challenge to science and engineering, A. V. Astin, SPE J. 17, No. 5, 455-458 (May 1961).

Low-temperature thermocouples I. Gold-cobalt or constantan versus copper or "normal" silver, R. L. Powell, M. D. Bunch, and R. J. Corruccini, Cryogenies 1, 139-150 (Mar. 1961).

Dielectric properties of polyamides, A. J. Curtis, J. Chem. Phys. 34, No. 5, 1849-1950 (May 1961).

Natural and synthetic rubbers: Review of test methods, F. J. Linnig, M. Tyron, and E. J. Parks, Anal. Chem. 33, 127R (Apr. 1961).

Magnetic susceptibility of tetratonal titanium dioxide, F. E. Senftle, T. Pankey, and F. A. Grant, Phys. Rev. 120, 820 (Nov. 1960).

Equipment and procedures for the evaluation of total hemispherical emittance, J. C. Richmond and W. N. Harrison, Bull. Am. Ceram. Soc. 39, No. 11, 668 (Nov. 1960).

Limitations of radiosonde punch-card records for radiometeorological studies, B. R. Bean and B. A. Cahoon, J. Geophys. Research 66, No. 1, 328-331 (Jan. 1961).

Evaluations of some oxide glasses for use in infrared material, G. W. Cleek and T. G. Scuderi, Proc. Infrared Information Symp. 5, No. 4 (Aug. 1960).

Improvement of some of the properties of sole leathers by impregnation with polymers, J. R. Kanagy, J. Am. Leather Chemists Assoc. LVI, No. 7, 322-342 (July 1961).

A transitor frequency meter, F. R. Bretemps and S. Saito, Electronic Inds. 19, No. 10, 196-198 (Oct. 1960).

Studies on the flame-spraying of aluminum oxide, D. G. Moore, Metal Finishing J. 6, No. 70, 397 (Oct. 1960).

Self-qualification of laboratories, A. T. McPherson, Instr. and Control Systems 34, No. 7, 1265 (July 1961).

Four-color achromats and superchromats, R. E. Stephens, J. Opt. Soc. Am. 50, No. 10, 1016-1019 (Oct. 1960).

Refining measurements by capacitance techniques, F. K Harris and R. D. Cutkosky, ISA-J. Instr. Soc. Am. 8, No. 2, 63-66 (Feb. 1961).

Standards for plastic pipe fittings, F. W. Reinhart, SPE J. 17, 160 (Feb. 1961).

Post Office mechanization, B. M. Levin, M. C. Stark, and P. C. Tosini, Elec. Engr. 80, 105-110 (Feb. 1961).

Comparison of observed tropospheric refraction with values computed from the surface refractivity, B. R. Bean, IRE Trans. Ant. Prop. AP-9, 415-416 (July 1961).

The precision measurement of transformer ratios, R. D. Cutkosky and J. Q. Shields, IRE Trans. Instrumentation I-9, No. 2, 243-250 (Sept. 1960).

Studies of environmental factors in a family-size underground shelter, P. R. Achenbach, F. J. J. Drapeau, and C. W. Phillips, Report OCDM-NBS-60-1 issued Office of Civil Defense Mobilization (Mar. 1961).

Electrodes for $p \mathrm{H}$ measurements, R. D. Bates, J. Electroanalytical Chem. 3, 93 (1961).

Phase-shift method for one-dimensional scattering, A. H Kahn, Am. J. Phys. 29, 77 (1961).

Electric field distribution in a dense plasma, J. L. Jackson, Phys. Fluids 3, 927-931 (1960).

A precision RF attenuation calibration system, C. M. Allred and C. C. Cook, PGI Trans. Instrumentation I-9, No. 2, 268-274 (Sept. 1960).

A vibrating sample magnetometer, N. V. Frederick, IRE Trans. Instrumentation I-9, No. 2, 194-196 (Sept. 1960). Standards development by ASTM committee D-20 on plastics, F. Reinhart, SPE J. 16, 800 (Aug. 1960).

A nuclear magnetic resonance study of any-anti isomerism in ketoximes, K. Lustig, J. Phys. Chem. 65, 491 (1961).

A standards current transformer and comparison methodA basis for establishing ratios of currents at audio frequencies, B. L. Dunfee, IRE Trans. Instrumentation I-9, No. 2, 231-236 (Sept. 1960).

Evaluating the freezing-and-thawing durability of concrete by laboratory tests in the U.S.A., H. T. Arni and R. L. Blaine, Preliminary Report on Symposium on Durability of Concrete of RILEM, Prague, Czechoslovakia, 218-231 (July 1961).

Spectroscopic standard samples of titanium and high-temperature alloys, R. E. Michaelis, J. Appl. Spectroscopy 15, 7 (1961).

Analytical laboratory problems, J. Mandel, Trans. Metropolitan Conf. Am. Soc. Quality Control, 99-107 (Feb. 1960.) 
Some experimental aspects of nuclear orientation, E. Ambler, Proc. 10th Inter. Congress of Refrigeration, Copenhagen, Denmark, 1959, Progress in Refrigeration in Science and Technology I, 195-193 (Pergamon Press, London, England 1960).

Absorption spectrum and magnetic properties of osmium hexafluoride, J. C. Eisenstein, J. Chem. Phys. 34, 310-318 (1961).

Rapid method for interpolating refractive index measurements, O. N. Stavroudis and L. E. Sutton, J. Opt. Soc. Am . 51, No. 3, 368-370 (Mar. 1961).

Comparison of four different methods of determining drying shrinkage of concrete masonry units, J. O. Bryson and D. Watstein, J. Am. Concrete Inst. 58, No. 2, 163-184 (Aug. 1961).

Effect of departures from local thermodynamic equilibrium on inferences on stellar atmospheric temperatures, R. N. Thomas, Book, Optical Spectrometric Measurements of High Temperatures, P. J. Dickerman, ed., 14-26 (University of Chicago Press, Chicago, Illinois, 1961).

The retannage of chrome-tanned leather with vegetable tannins, J. R. Kanagy, J. Am. Leather Chemists Assoc. IVVI, No. 6, 252-272 (June 1961).

Improved electron filter lens, J. A. Simpson and L. Marton, Rev. Sci. Instr. 32, No. 7, 802-803 (July 1961).

Static and dynamic calibrations of pressure measuring instruments at the National Bureau of Standards, E. C. Lloyd and D. P. Johnson, Automatic and Remote Control Proc. 1st Intern. Congress of IFAC, 274-280 (1960).

X-ray diffraction study of accellular telecost bone, M. L. Moss and A. S. Posner, Nature 188, 1037 (Dec. 1960).

The NBS absolute gravity experiment, D. R. Tate, Proc. 2d Air Force Cambridge Research Center Military Geodesy Seminar, GRD Research Notes, 40 (Jan. 12-14, 1960).

Adsorption of polyesters and other polymers on glass and other substrates, R. R. Stromberg and G. M. Kline, Modern Plastics 38, 123 (Apr. 1961); 38, 241 (May 1961); Poliplastic 8, 15 (Jan.-Feb. 1961).

Tunnel diode large-signal simulation study, S. B. Geller and P. A. Mantek, Proc. IRE 49, No. 4, 803 (Apr. 1961).

Thermal degradation of organic polymers, S. L. Madorsky, Polymer Sci. 17, No. 7 (July 1961).

Determination of carboxyl in cellulose - comparison of various methods, W. K. Wilson and J. Mandel, Tappi 44, No. 2, 131 (1961)

A recalibration of NBS carbon-14 standards by Geiger-Muller and proportional gas counting, W. B. Mann, H. H. Seliger, W. F. Marlow, and R. W. Medlock, Rev. Sci. Instr. 31, No. $7,690(1960)$.

Computer for weather data acquisition, F. Meissner, J. Cunningham, and C. Kettering, Proc. Eastern Joint Computer Conf. 18, No. 2.1, 57-66 (Dec. 1960)

The solar spectrum from 2635 to 2085 A, H. H. Malitson, J. D. Purcell, R. Tousey, and C. E. Moore, Astrophys. J. 132, 746 (1960).

Winter thermal radiation studies in Yellowstone Park, D. M. Gates, Science 134, 32-35 (July 7, 1961).

Vapor phase $\gamma$-radiolysis of acetone, L. J. Stief and P. Ausloos, J. Phys. Chem. 65, 877 (1961).

Amine buffers for $p \mathrm{H}$ control, R. G. Bates, Ann. N. Y. Acad. Sci. 92, 341 (1961).

Cryogenic adhesive properties of bisphenol- $A$ epoxy resins, M. J. Hiza and P. L. Barrick, SPE Trans. 1, 73-79 (Apr. 1961).

Infrared spectra of solid hydrocarbons at very low temperatures, J. J. Comeford and J. H. Gould, J. Mole. Spectroscopy 5, 474 (1960).

Recent work with compensated internal gas counters for the standardization of gaseous radionuclides, W. B. Mann, Proc. Symp. Metrology of Radioactive, IAEA, Vienna, 307 (1960).

Vanadium oxytrichloride [Vanadyl(v) chloride], R. B. Johannesen, Book, Inorganic Syntheses, E. C. Rochow, Editor-in-Chief (MeGraw-Hill Book Co., New York, N.Y.), IV. Chapt 5B, No. 38, 119-120 (1960).

Concerning the potential refractive index and the molecular refractivity, B. R. Bean and J. D. Horn, J. Meteorol. 18, No. 3, 427-428 (June 1961).

Standard samples and related materials for spectrochemical analysis, R. E. Michaelis, Am. Soc. Testing Materials Spec. Tech. Publ. No. 58-D, 116 (1960).

Aromatic fluorocarbons: Thermal stability and synthesis, L. A. Wall, Proc. 6th Joint Army-Navy-Air Force Elastomer Conf., Oct. 18-20, 1960, I, 150 (U.S. Army Quartermaster Research \& Engineering Command, Natick, Mass.).

The ionization constant of hydroxylamine, R. A. Robinson and V. E. Bower, J. Phys. Chem. 65, 1279-1280 (1961).

Multiple layer insulation for cryogenic applications, R. H. Kropschot, Cryogenics 1, 171-177 (Mar. 1961).

Extinguishment effectiveness of some powdered materials on hydrocarbon fires, T. G. Lee and A. F. Robertson, Fire Research Abstracts and Reviews, Natl. Acad. Sci.-Natl. Research Council Publ. $\mathbf{8 8 6 ,}$, June 1961).

Forty years of dental research at the National Bureau of Standards, W. T. Sweeney, Capitol Chemist 10, 228 (Oct. 1960).

A stainless steel for standard weights, S. J. Rosenberg and T. P. Royston, Materials Research \& Standards (ASTM Bull.) 1, No. 1, 21 (Jan. 1961).

Long-term working stress of thermoplastic pipe, F. W. Reinhart, SPE J., 751-754 (Aug. 1961).

NBS atomic frequency standards, R. C. Mockler and R. E. Beehler, Proc. 14th Annual Symp. Frequency Control, Atlantic City, N.J., May 31-June 3, 1960, p. 298-309 (Signal Corps Eng. Laboratories, Fort Monmouth, N.J., 1961).

Microwave Zeeman effect of free hydroxyl radicals: ${ }^{2} \mathrm{II}_{1 / 2}$ levels, H. E. Radford, Phys. Rev. 122, 114 (1961).

A precision RF power transfer standards, P. A. Hudson, IRE Trans. Instrumentation I-9, 280-283 (Sept. 1960).

The fluorescence and phosphorescence of trifluoroacetons vapor, P. Ausloos and E. Murad, J. Am. Chem. Soc. 83, 1327 (1961).

The morphology of sporadic E, E. K. Smith, Jr., Proc. XIIIth Gen. Assembly URSI (London, England, 1960), Natl. Acad. Sci.-Natl. Research Council Publ., Report No. 880, 218 (1961)

How to determine stack load limits, R. S. Wyly, Contractor, pp. 19-20 (Aug. 15, 1961).

$\gamma$-Irradiation of liquid and solid oxygen, D. W. Brown and L. A. Wall, Phys. Chem. 65, 915 (1961).

Testing of ball bearings with five different separator materials at $9200 \mathrm{RPM}$ in liquid nitrogen, J. A. Brennan, W. A. Wilson, R. Radebaugh, and B. W. Birmingham, Am. Soc. Mech. Engrs., No. 61-LUBS-18, 1-8 (Apr. 1961).

The CRPL electron density profile program: Some features and early results, J. W. Wright, Proc. URSI-AGI Symp. (Brussels, Belgium, 1959), Book, Some Ionospheric Results Obtained During IGY, Ed. W. J. G. Beyon (Elsevier Publ. Co., Princeton, N.J.), 215 (1960).

Kinetic isotope effects in the reaction of methyl radicals with ethane, $-d_{6}$ and ethane-1, 1, 1- $\mathrm{d}_{3}$, J. R. McNesby, J. Phys. Chem. 64, No. 11, 1671 (Nov. 1960).

Digest of "A general description of D-C digital voltmeters," C. Stansbury, AIEE Trans. Paper 61-721 (Apr. 1961).

Nomenclature of carbohydrates, R. S. Tipson, J. Chem. Documentation 2, No. 3, 3-7 (1961).

Determination of wax in paper, W. L. Marrow, Tappi 44, No. 2, 120 (1961).

Mortar for cavity walls, C. C. Fishburn, Building Research Institute, 1960 Spring Conf. Program on Insulated Cavity Walls, Natl. Acad. Sci.-Natl. Research Council Publ. 793, 37 (1960).

Variation of [OI] $5577 \AA$ emission in the upper atmosphere, F. E. Roach, Ann. Geophys. 17, 172-180 (Apr.-June 1961). Continuously operating $\mathrm{He}^{3}$ refrigerator for producing temperature down to $1 / 4^{\circ} \mathrm{K}$, E. Ambler and R. E. Dove, Rev. Sci. Instr. 32, No. 6, 737-739 (June 1961).

Location of the plane of best average definition for airplane camera lenses, F. E. Washer and W. P. Tayman, Photogrammetric Eng. 26, No. 3, 475-488 (1960).

Low temperature properties of cerous magnesium nitrate, R. P. Hudson, R. S. Kaeser, and H. E. Radford, Proc. VIIth Intern. Conf. Low Temperature Physics, University of Toronto, Canada, p. 45-48 (1960).

The oxide films formed on copper single crystal surfaces in water II. Rate of growth at room temperature, J. Kruger, J. Electrochem. Soc. 108, No. 6, 503 (June 1961). 
A review of the hydraulics of circular sewers in accordance with the Manning formula, R. S. Wyly, Yearbook of Am.

F Soc. of Sanitary Engineering 38, 154 (1960).

Line shape of ultraviolet absorption in solid noble gases, P. H. E. Meijer, J. Chem. Phys. 34, 2078-2082 (1961).

A screening method for large information retrieval systems, R. T. Moore, Proc. Western Joint IRE-AIEE-ACM Computer Conf., Los Angeles, Calif. 6.3, 259-274 (1961).

Humidity standards, A. Wexler, Tappi 44, No. 6, 180a (June 1961).

Ionospheric "forward" scattering, D. K. Bailey, Proc. XIIIth Gen. Assembly of URSI (London, England, 1960), Natl. Acad. Sci.-Natl. Research Council Publ. Report No. 880, 281 (1961)

Apparatus for controlled slack quenching, N. L. Carwile, M. R. Meyerson, and S. J. Rosenberg, Materials Research and Standards (ASTM Bull.) 1, No. 7, 532-536 (July 1961).

Exponential temperature dependence of Young's modulus for several oxides, J. B. Watchman, Jr., W. E. Tefft, D. G. Lam, Jr., and C. S. Apstein, Phys. Rev. 122, 1754 (June 1961).

A high-resolution ammonia-maser-spectrum analyzer, J. A. Barnes and L. E. Heim, IRE Trans. Instrumentation I-10, 4-8 (June 1961).

Electron spin resonance spectra of free-radical intermediates formed by reaction of polystyrene with atoms of hydrogen and deuterium, R. B. Ingalls and L. A. Wall, J. Chem. Phys. 35, No. 1, 370-371 (July 1961).

The X- or gamma-ray energy absorption of transfer coefficient: Tabulations and discussion, R. T. Berger, Radiation Research 15, No. 1, 1-29 (July 1961).

Reinhart, F. M., Twenty-year atmospheric corrosion investigation of zinc-coated and uncoated wire and wire products, Am. Soc. Testing Materials Spec. Tech. Publ. 290, 1-141 (June 1961).

Time standards, A. G. MeNish, Instr. and Control Systems 33, 1340-1341 (Aug. 1960).

Adsorption of nitrogen and argon on mineralogical graphite and diamond at 77 and $90^{\circ} \mathrm{K}$, J. de Dios Lopes-Gonzales, F. G. Carpenter, and V. R. Deitz, J. Phys. Chem. 65, No. 7 , 1112-19 (July 1961)

Absorption of carbon dioxide by solutions of 2-amino-2(hydroxymethyl)-1, 3-propanediol, R. G. Bates and H. B. Hetzer, Anal. Chem. 33, No. 9, 1285 (Aug. 1961).

Stress-strain relationships in yarns subjected to rapid impac loading; Part 7. Stress-strain curves and breaking-energy data for textile yarns, J. C. Smith, P. J. Shouse, J. M. Blandford, and K. M. Towne, Textile Research J. 31, No. 8, 721-734 (Aug. 1961)

V. Mutual interference between surfaces and satellite communication systems, B. Hartman, M. T. Decker, Chapt. 5, p. 111-157, Book, Frequency Allocations for Space Communications (Report of the Joint Tech. Adv. Comm. of IRE-EIA to the Federal Communications Commission, Mar. 1961).

Quantum theory of interference effects in the mixing of light from phase-independent sources, U. Fano, Am. J. Phys. 29, No. 8, 539-545 (August 1961).

Heats and volumes of mixing in several $\mathrm{C}_{1}$, hydrocarbon systems, R. S. Jessup and C. L. Stanley, J. Chem. Eng. Data 6, No. 3, 368-371 (July 1961).

Some observations of copper deposits on single crystals of copper, I. Giron and F. Ogburn, J. Electrochem. Soc. 108, No. 9, 842-846 (Sept. 1961).

Slow drift solar radio bursts: harmonic frequency ratios, solar longitude dependence, and frequency drift rates, M. B. Wood, Australian J. Phys. 14, No. 2, 234-241 (1961).

Infrared spectrum and structures of the $\mathrm{NF}_{2}$ radical, $\mathrm{M}$. D Harmony, R. J. Myers, L. J. Schoen, D. R. Lide, Jr., and D. E. Mann, J. Chem. Phys. 35, No. 3, 1129 (Sept. 1961).

Fibrous silica, W. Haller, Nature 191, No. 4789, 662-663 (Aug. 12, 1961)

Control terminology-A report on U.S. standards activity, H. L. Mason, Control Eng. 8, No. 10, 67-70 (Oct. 1961).

Behavior and evaluation of rubber, R. D. Stiehler, Am. Concrete Pipe Assoc. Tech. Memo. (Oct. 1961).
Welded butt joints with fine wires, L. Martz, Rev. Sci. Instr., 32, No. 8, 990-991 (Aug. 1961).

Systematic errors in physical constants, W. J. Youden, Physics Today 14, No. 9, 32-34, 36, 38, 40, 42 (Sept. 1961).

Mass spectrometric study of the thermal dissociation of $\mathrm{N}_{2} \mathrm{~F}_{4}$, J. T. Herron and V. H. Dibeler, J. Chem. Phys. 35, No. 2, 747-748 (Aug. 1961).

A study of solar activity associated with polar cap absorption, (abstract), C. S. Warwick and M. B. Wood. Polar Cap Absorption Conf. (Kiruna, Sweden, Aug. 8-11, 1960), Arkiv Geofyski 3, No. 21, 457 (1960).

Binary oxide systems, H. R. Hoekstra and H. S. Parker, Chapt. 6, sect. 6, p. 271-304, Book, $\mathrm{UO}_{2}$, Properties and Nuclear Application (U.S. Superintendent of Documents, Government Printing Office, Washington 25, D.C., Aug. 1961).

Wind resistance of asphalt shingle roofing, W. C. Cullen, Natl. Acad. Sci.-Natl. Research Council, 33-42 (1961).

Pulse voltage comparator measures height of positive or negative pulses, O. B. Laug, Electronics 34, No. 36, 70-71 (Sept. 1961).

Spectroscopic observations of the stratosphere and mesosphere, D. M. Gates, Instr. Soc. Am. J. Conf. Preprint No. 2-NY60 (Sept. 1960).

Supercooling in the barnyard, C. P. Saylor, Capital Chemist 10, No. 9, 292-293 (Dec. 1960).

Low-angle X-ray diffraction of crystalline nonoriented polyethylene and its relation to crystallization mechanisms, L. Mandelkern, A. S. Posner, A. F. Diorio, and D. E. Roberts, J. Appl. Phys. 32, No. 8, 1509-1517 (Áug. 1961).

References to contemporary papers on acoustics, W. Koidan, Acoust. Soc. Am. 33, No. 5, 705-716 (May 1961).

Ultraviolet absorption spectrum of ammonia in solid argon at $4.2^{\circ}$ K, K. Dressler, J. Chem. Phys. 35, No. 1, 165-169 (July 1961).

Photon-dissociation of water: initial nonequilibrium populations of rotational states of $\mathrm{OH}\left({ }^{2} \Sigma^{+}\right)$, I. Tanaka, T. Carrington, and H. P. Broida, J. Chem. Phys. 35, No. 2 , 750-751 (Aug. 1961).

Electrical conduction in $p$-type titanium sesquioxide, J. Yahia and H. P. R. Frederikse, Phys. Rev. 123, No. 4 1257-1261 (Aug. 15, 1961).

Parameters $\alpha$ and $\beta$ in the spectra of the iron group. R. E. Trees and C. K. Jorgensen, Phys. Rev. 132, No. 4, 1278 1280 (Aug. 15, 1961).

$\mathrm{Kr}^{96}$ and atomic-beam-emitted $\mathrm{Hg}^{198}$ wavelengths, $\mathrm{R}$. L. Barger, and K. G. Kessler, J. Opt. Soc. Am. 51, No. 8, 827-829 (Aug. 1961).

Excess noise in microwave detector diodes, J. J. Faris and J. M. Richardson, IRE Trans. Microwave Theory and Tech. MTT-9, No. 4, 312-314 (July 1961).

Conductance of solutions of water, acetic anhydride, and acetyl chloride in acetic acid, T. B. Hoover and A. W. Hutchinson, J. Am. Chem. Soc. 83, No. 16, 3400-3405 (1961).

Effect of branching on the thermal decomposition of polymers, L. A. Wall, Soc. Chem. Ind. Mono. 13, p. 146-162 (Page Bros. (Norwich) Ltd., England, 1961).

Phase equilibria research in systems involving the rare earth oxides, R. S. Roth, Book, Rare Earth Research, Pt. II, pp. 88-95 (The MacMillan Company, New York, N.Y., 1961).

Degradation of poly (2, 3, 4, 5, 6-pentafluorostyrene), L. A. Wall, J. M. Antonucci, S. Straus, and M. Tryon, Soc. Chem. Ind. Mono. No. 13, 295-302 (1961).

*Publications for which a price is indicated (except for Technical Notes) are available only from the Superintendent of Documents, U.S. Government Printing Office, Washington 25, D.C. (foreign postage, one-fourth additional). Technical Notes are available only from the Office of Technical Services, U.S. Department of Commerce, Washington 25, D.C. (Order by $P B$ number). Reprints from outside journals and the NBS Journal of Research may often be obtained directly from the authors. 